\title{
Synthesis, Molecular Docking Studies, and Antifungal Activity Evaluation of New Benzimidazole-Triazoles as Potential Lanosterol 14 $\alpha$-Demethylase Inhibitors
}

\author{
Nafiz Öncü Can,, ${ }^{1,2}$ Ulviye Acar Çevik, ${ }^{2,3}$ Begüm Nurpelin Sağlık, ${ }^{2,3}$ Serkan Levent,, ${ }^{2,3}$ \\ Büşra Korkut, ${ }^{4}$ Yusuf Özkay, ${ }^{2,3}$ Zafer Asım Kaplancıklı, $^{2}$ and Ali Savaş Koparal ${ }^{5}$ \\ ${ }^{1}$ Department of Analytical Chemistry, Faculty of Pharmacy, Anadolu University, 26470 Eskişehir, Turkey \\ ${ }^{2}$ Department of Pharmaceutical Chemistry, Faculty of Pharmacy, Anadolu University, 26470 Eskişehir, Turkey \\ ${ }^{3}$ Doping and Narcotic Compounds Analysis Laboratory, Faculty of Pharmacy, Anadolu University, 26470 Eskişehir, Turkey \\ ${ }^{4}$ Department of Pharmaceutical Toxicology, Faculty of Pharmacy, Anadolu University, 26470 Eskişehir, Turkey \\ ${ }^{5}$ Department of Environmental Engineering, Faculty of Engineering, Anadolu University, Eskişehir, Turkey \\ Correspondence should be addressed to Yusuf Özkay; yozkay@anadolu.edu.tr
}

Received 8 August 2017; Accepted 9 October 2017; Published 12 December 2017

Academic Editor: Gabriel Navarrete-Vazquez

Copyright (C) 2017 Nafiz Öncü Can et al. This is an open access article distributed under the Creative Commons Attribution License, which permits unrestricted use, distribution, and reproduction in any medium, provided the original work is properly cited.

\begin{abstract}
Due to anticandidal importance of azole compounds, a new series of benzimidazole-triazole derivatives (5a-5s) were designed and synthesized as ergosterol inhibitors. The chemical structures of the target compounds were characterized by spectroscopic methods. The final compounds were screened for antifungal activity against Candida glabrata (ATCC 90030), Candida krusei (ATCC 6258), Candida parapsilosis (ATCC 22019), and Candida albicans (ATCC 24433). Compounds 5i and 5s exhibited significant inhibitory activity against Candida strains with $\mathrm{MIC}_{50}$ values ranging from 0.78 to $1.56 \mu \mathrm{g} / \mathrm{mL}$. Cytotoxicity results revealed that $\mathrm{IC}_{50}$ values of compounds $\mathbf{5 i}$ and $\mathbf{5 s}$ against NIH/3T3 are significantly higher than their MIC $_{50}$ values. Effect of the compounds $\mathbf{5 i}$ and $\mathbf{5 s}$ against ergosterol biosynthesis was determined by LC-MS-MS analysis. Both compounds caused a significant decrease in the ergosterol level. The molecular docking studies were performed to investigate the interaction modes between the compounds and active site of lanosterol 14- $\alpha$-demethylase (CYP51), which is as a target enzyme for anticandidal azoles. Theoretical ADME predictions were also calculated for final compounds.
\end{abstract}

\section{Introduction}

Recently, the incidence of systemic fungal infection has become an important complication and a major cause of morbidity and mortality in immune compromised individuals such as patients undergoing anticancer chemotherapy or organ transplants and patients with AIDS [1,2]. According to the mechanism of action, there are six classes of antifungal agents: fungal ergosterol synthesis inhibitors (azoles: ketoconazole, fluconazole, and voriconazole), glucan synthesis inhibitors (echinocandins and caspofungin), ergosterol disruptors (polyenes antibiotics: amphotericin B), squalene epoxidase inhibitors (terbinafine and naftifine), chitin synthesis inhibitors (nikkomycin), and nucleic acid synthesis inhibitors (5-fluorocytosine) (Figure 1) [3]. Due to its high therapeutic index, azoles are first-line drugs for the treatment of invasive fungal infections $[4,5]$. Most therapies, designed to treat fungal infections, target the ergosterol biosynthesis pathway or its end product [6].

In fungi, lanosterol 14- $\alpha$-demethylase (CYP51) belongs to a superfamily of monooxygenases called cytochrome P450, which catalyzes the oxidative removal of the 14-methyl group (C-32) of lanosterol to give 14,15-desaturated intermediates in ergosterol biosynthesis. CYP51 is one of the key enzymes of sterol biosynthesis in different biological kingdoms and serves the metabolic function such as membrane permeability, membrane fluidity, enzyme activity, cell morphology, and cell cycle progression [7-9]. This enzyme is found in all 


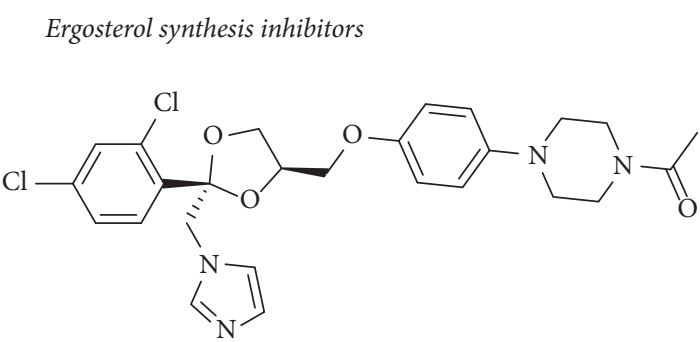

Ketoconazole<smiles>OC(Cn1cncn1)(Cn1cncn1)c1ccc(F)cc1F</smiles>

Fluconazole<smiles>C[C@H](c1ncncc1F)[C@@](C)(Cn1cncn1)c1ccc(F)cc1F</smiles>

Voriconazole

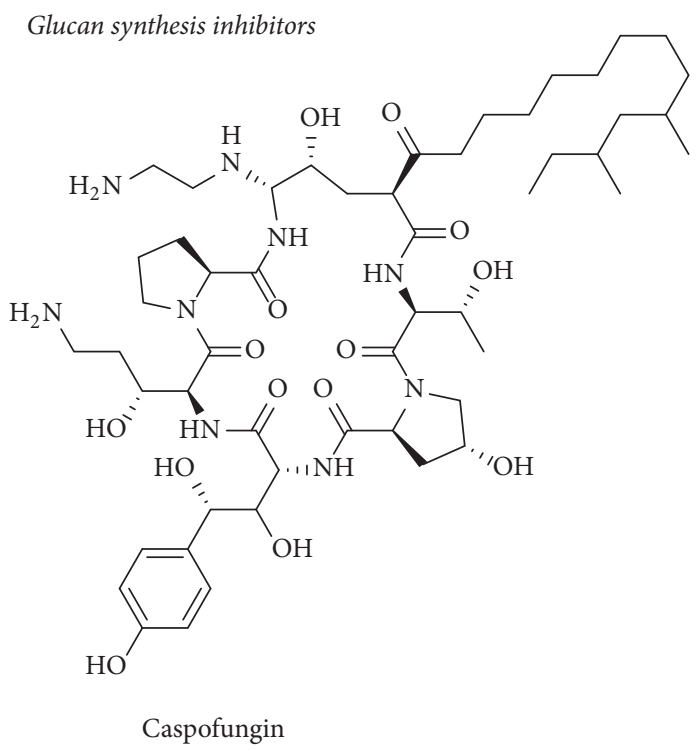

Ergosterol disruptor

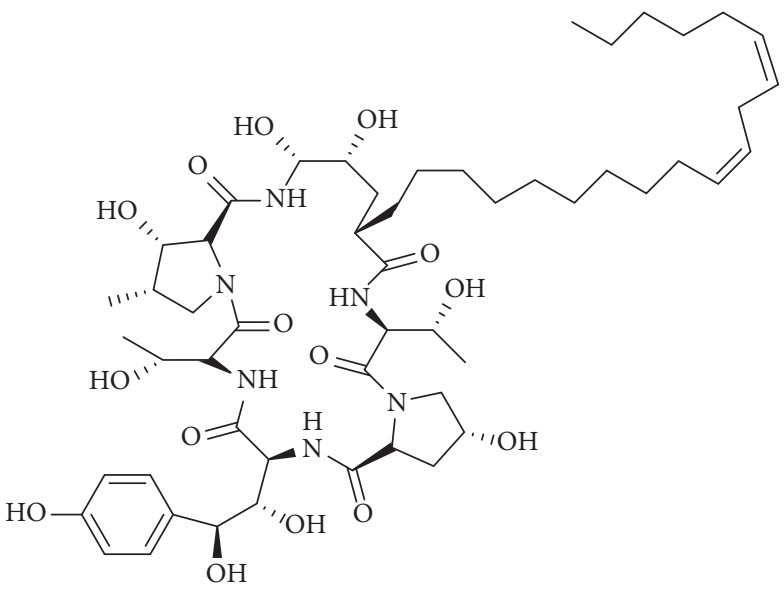

Echinocandins

Chitin synthesis inhibitors<smiles>C[C@@H]([C@H](N)C(=O)N[C@H](C(=O)O)[C@H]1O[C@@H](n2ccc(=O)[nH]c2=O)[C@H](O)[C@@H]1O)[C@H](O)c1ccc(O)cn1</smiles>

Nikkomycin

Nucleic acid synthesis inhibitors

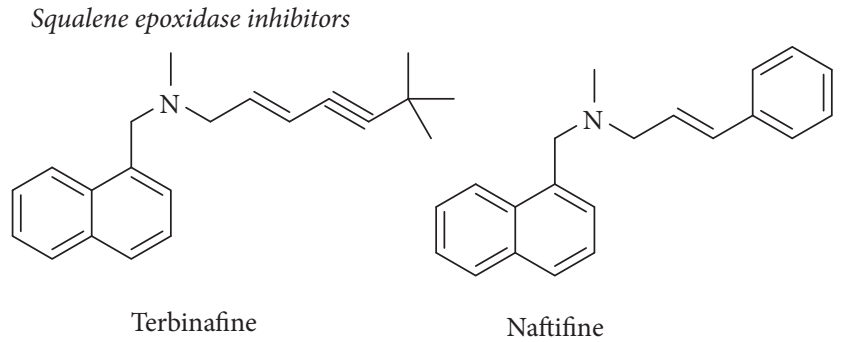<smiles>Nc1nc(=O)[nH]cc1F</smiles>

5-Fluorocytosine

FIGURE 1: Chemical structures of some antifungal drugs which are in clinical use. 
eukaryotes (including humans) and because the azoles interact also with other cytochrome P450-dependent enzymes (CYP3A4), a selective inhibition of the enzyme is essential for an increased therapeutic index [10-12].

In general, the active site of CYP51 is divided into four subsites: a coordination bond with iron of the heme group, the hydrophobic region, the hydrophilic H-bonding region, and the narrow hydrophobic cleft formed. The affinity of azole antifungals to the lanosterol 14a-demethylase is determined not only by the coordination binding of the nitrogen of azole ring to the heme iron in the active side (N-4 of triazole and $\mathrm{N}-3$ of imidazole) but also by the affinity of N-1 substituent for the apoprotein part of the enzyme. The remaining part of the azole antifungal fits in the similar way like lanosterol in the hydrophobic groove of lanosterol 14- $\alpha$-demethylase [13-17].

Based on these properties of the active site of CYP51, we are provided with an attempt to design new azole derivatives to find potent systemic antifungal agents that have a broad antifungal spectrum but with less potential to develop drug resistance. Several studies have been performed, and many of them have supported the finding that benzimidazole and triazole rings were significant structural moieties for antifungal activity [18-28]. In the present study, prompted from anticandidal potential of azole compounds, new benzimidazoletriazole hybrid compounds were synthesized and evaluated for antifungal activity.

\section{Experimental}

2.1. Chemistry. Entire chemicals used in the study were purchased from either Sigma-Aldrich (Sigma-Aldrich Corp., St. Louis, MO, USA) or Merck (Merck KGaA, Darmstadt, Germany) and used without further chemical or biological purification. Microwave syntheses were realized by using a Monowave 300 high-performance microwave reactor (Anton-Paar, Austria). Melting points of synthesized compounds were determined by using an automatic melting point determination system (MP90 series, MettlerToledo, OH, USA) and were presented as uncorrected. ${ }^{1} \mathrm{H}$ and ${ }^{13} \mathrm{C}$ NMR spectra were recorded in DMSO- $d_{6}$ by a Bruker digital FT-NMR spectrometer (Bruker Bioscience, MA, USA) at $300 \mathrm{MHz}$ and $75 \mathrm{MHz}$, respectively. The IR spectra of the compounds were recorded using an IRAffinity$1 S$ Fourier transform IR (FTIR) spectrometer (Shimadzu, Kyoto, Japan). High resolution mass spectrometric studies were performed using an LCMS-IT-TOF system (Shimadzu, Kyoto, Japan). Chemical purities of the compounds were checked by classical TLC applications performed on silica gel $60 \mathrm{~F}_{254}$ (Merck KGaA, Darmstadt, Germany); LCMS-IT-TOF chromatograms were also used for the same purpose.

2.1.1. Methyl4-(5(6)-Methyl-1H-benzimidazol-2-yl)benzoate (1). In a vial $(30 \mathrm{~mL})$ of microwave synthesis reactor (AntonPaar, Monowave 300, Austria) equipped with a magnetic stirrer, a solution of methyl-4-formyl benzoate $(2.4 \mathrm{~g}, 0.015 \mathrm{~mol})$ in DMF $(10 \mathrm{~mL})$ and sodium disulfite $(2.85 \mathrm{~g}, 0.015 \mathrm{~mol})$ were heated under conditions of $240^{\circ} \mathrm{C}$ and 10 bar for $5 \mathrm{~min}$. After this stage, 5-methyl-1,2-phenylenediamine (1.83 g, $0.015 \mathrm{~mol}$ ) was added and then reaction mixture was kept under the same reaction conditions in microwave reactor. The mixture was cooled and poured into iced-water. The obtained solid was filtered, washed with water, dried, and recrystallized from ethanol to gain product with $86 \%$ yields.

2.1.2. 4-(5(6)-Methyl-1H-benzimidazol-2-yl)benzohydrazide (2). Methyl 4-(5-methyl-1H-benzimidazol-2-yl)benzoate (1) $(2.66 \mathrm{~g}, 0.01 \mathrm{~mol})$ and hydrazine hydrate $(5 \mathrm{~mL})$ in ethanol $(15 \mathrm{~mL})$ were heated under the conditions of $150^{\circ} \mathrm{C}$ and 10 bar for $10 \mathrm{~min}$ in microwave synthesis reactor (Anton-Paar Monowave 300, Austria). The mixture was cooled and poured into iced-water. The obtained solid was filtered, washed with water, dried, and recrystallized from ethanol to gain product with $92 \%$ yields.

2.1.3. N-Alkyl-2-[4-(5(6)-methyl-1H-benzimidazol-2-yl)benzoyl]hydrazine-1-carbothioamide (3a, 3b). 4-(5-Methyl-1Hbenzimidazol-2-yl)benzohydrazide (2) $(2.66 \mathrm{~g}, 0.01 \mathrm{~mol})$ and methyl isothiocyanate or ethyl isothiocyanate $(0.012 \mathrm{~mol})$ in ethanol were refluxed for $2 \mathrm{~h}$. The precipitated product was filtered, washed with ethanol, and dried. Compounds $\mathbf{3 a}$ and 3b were obtained with yields of $78 \%$ and $81 \%$, respectively.

2.1.4. 4-Alkyl-5-[4-(5(6)-methyl-1H-benzimidazol-2-yl)phenyl)]-4H-1,2,4-triazole-3-thiol (4a-4b). N-Alkyl-2-[4-(5(6)methyl-1H-benzimidazol-2-yl)benzoyl]hydrazine-1-carbothioamide $(3 \mathbf{a}, 3 \mathbf{b})(0.001 \mathrm{~mol})$ in ethanol was refluxed under stirring for $2 \mathrm{~h}$ in the presence of $\mathrm{NaOH}(0.012 \mathrm{~mol})$. After completion of reaction, the solution was acidified with $\mathrm{HCl}$ $37 \%$; the precipitate was filtered, washed with water, dried, and then recrystallized from ethanol. Compounds $\mathbf{4 a}$ and $\mathbf{4 b}$ were obtained with yields of $71 \%$ and $73 \%$, respectively.

2.1.5. 2-(4-(4-Alkyl-5-(2-(substitutedphenyl)-2-oxo-ethylthio)4H-1,2,4-triazol-3yl)-phenyl)-5(6)-methyl-1H-benzimidazole $(5 \boldsymbol{a}-5 \mathrm{~s})$. A solution of $4 \mathbf{a}$ or $4 \mathbf{b}(0.001 \mathrm{~mol})$ in acetone $(10 \mathrm{~mL})$, an appropriate substituted 2-bromoacetophenone derivative $(0.001 \mathrm{~mol})$, and potassium carbonate $(0.138 \mathrm{~g}$, $0.001 \mathrm{~mol}$ ) were refluxed at $40^{\circ} \mathrm{C}$ for $12 \mathrm{~h}$. The solvent was evaporated; residue was washed with water, dried, and recrystallized from ethanol.

2.1.6. 2-(4-(4-Methyl-5-(2-phenyl-2-oxo-ethylthio)-4H-1,2,4triazol-3yl)-phenyl)-5(6)-methyl-1H-benzimidazole (5a). Yield: $81 \%$. M.p. $247-248^{\circ}$ C. FTIR (ATR, $\left.\mathrm{cm}^{-1}\right)$ : $3211(\mathrm{~N}-\mathrm{H})$, $1676(\mathrm{C}=\mathrm{O}), 848$ (1,4-disubstituted benzene $\mathrm{C}-\mathrm{H}) .{ }^{1} \mathrm{H}-\mathrm{NMR}$ $\left(300 \mathrm{MHz}, \mathrm{DMSO}-d_{6}\right): \delta=2.44\left(3 \mathrm{H}, \mathrm{s},-\mathrm{CH}_{3}\right), 3.72(3 \mathrm{H}, \mathrm{s}$, $\left.-\mathrm{CH}_{3}\right), 4.96\left(2 \mathrm{H}, \mathrm{s},-\mathrm{CH}_{2}-\right), 7.07(1 \mathrm{H}, \mathrm{d}, J=8.19 \mathrm{~Hz}, \mathrm{Benz}-$ imidazole C-H), 7.42 ( $1 \mathrm{H}$, s, Benzimidazole C-H), 7.51-7.60 $(3 \mathrm{H}, \mathrm{m}$, Benzimidazole C-H, Monosubstituted Benzene C$\mathrm{H}), 7.70(1 \mathrm{H}, \mathrm{t}, J=7.88 \mathrm{~Hz}$, Monosubstituted Benzene C-H), $7.89(2 \mathrm{H}, \mathrm{d}, J=8.35 \mathrm{~Hz}, 1,4$-disubstituted benzene C-H), 8.04 $(2 \mathrm{H}, \mathrm{d}, \mathrm{J}=7.88 \mathrm{~Hz}$, Monosubstituted Benzene C-H), 8.32 $(2 \mathrm{H}, \mathrm{d}, J=8.35 \mathrm{~Hz}, 1,4$-disubstituted benzene $\mathrm{C}-\mathrm{H}), 13.15(1 \mathrm{H}$, $\mathrm{s}$, Benzimidazole -NH). ${ }^{13} \mathrm{C}-\mathrm{NMR}\left(75 \mathrm{MHz}, \mathrm{DMSO}-d_{6}\right): \delta$ (ppm): 21.81, 32.49, 41.27, 115.76, 124.54, 126.83, 127.18, 128.42, $128.93,129.22,129.31,130.37,131.62,132.37,134.26,135.73$, 150.37, 151.08, 155.30, 192.85. $[\mathrm{M}+\mathrm{H}]^{+}$calcd for $\mathrm{C}_{25} \mathrm{H}_{21} \mathrm{~N}_{5} \mathrm{OS}$ : 440.1540; found: 440.1527 . 
2.1.7. 2-(4-(4-Methyl-5-(2-(4-cyanophenyl)-2-oxo-ethylthio)4H-1,2,4-triazol-3yl)-phenyl)-5(6)-methyl-1H-benzoimidazole (5b). Yield: $83 \%$. M.p. $239-240^{\circ}$ C. FTIR (ATR, $\mathrm{cm}^{-1}$ ): 3093 $(\mathrm{N}-\mathrm{H}), 1680(\mathrm{C}=\mathrm{O}), 849$ (1,4-disubstituted benzene $\mathrm{C}-\mathrm{H})$. ${ }^{1} \mathrm{H}-\mathrm{NMR}\left(300 \mathrm{MHz}, \mathrm{DMSO}-d_{6}\right): \delta=2.49\left(3 \mathrm{H}, \mathrm{s},-\mathrm{CH}_{3}\right), 3.74$ $\left(3 \mathrm{H}, \mathrm{s},-\mathrm{CH}_{3}\right), 4.99\left(2 \mathrm{H}, \mathrm{s},-\mathrm{CH}_{2}-\right), 7.29(1 \mathrm{H}, \mathrm{d}, J=8.28 \mathrm{~Hz}$, Benzimidazole C-H), 7.57 (1H, s, Benzimidazole C-H), 7.67 $(1 \mathrm{H}, \mathrm{d}, J=8.28 \mathrm{~Hz}$, Benzimidazole $\mathrm{C}-\mathrm{H}), 8.02(2 \mathrm{H}, \mathrm{d}, J=$ $8.45 \mathrm{~Hz}, 1,4$-disubstituted benzene $\mathrm{C}-\mathrm{H}), 8.06(2 \mathrm{H}, \mathrm{d}, J=8.40$, 4-cyanophenyl C-H), 8.19 (2H, d, $J=8.40$, 4-cyanophenyl $\mathrm{C}-\mathrm{H}), 8.36(2 \mathrm{H}, \mathrm{d}, J=8.45 \mathrm{~Hz}, 1,4$-disubstituted benzene $\mathrm{C}-\mathrm{H}), 13.32\left(1 \mathrm{H}, \mathrm{s}\right.$, Benzimidazole -NH). ${ }^{13} \mathrm{C}-\mathrm{NMR}(75 \mathrm{MHz}$, DMSO- $\left.d_{6}\right): \delta$ (ppm): $21.73,32.59,41.13,114.31,114.84,116.03$, $118.56,126.77,127.59,128.17,129.47,129.54,130.16,133.32$, $135.06,135.36,139.04,149.01,151.26,154.96,193.43 .[\mathrm{M}+\mathrm{H}]^{+}$ calcd for $\mathrm{C}_{26} \mathrm{H}_{20} \mathrm{~N}_{6}$ OS: 465.1492; found: 465.1485 .

2.1.8. 2-(4-(4-Methyl-5-(2-(4-fluorophenyl)-2-oxo-ethylthio)4H-1,2,4-triazol-3yl)-phenyl)-5(6)-methyl-1H-benzimidazole (5c). Yield: $77 \%$. M.p. $255-256^{\circ}$ C. FTIR (ATR, $\mathrm{cm}^{-1}$ ): 2968 $(\mathrm{N}-\mathrm{H}), 1681(\mathrm{C}=\mathrm{O}), 843$ (1,4-disubstituted benzene $\mathrm{C}-\mathrm{H})$. ${ }^{1} \mathrm{H}-\mathrm{NMR}\left(300 \mathrm{MHz}\right.$, DMSO- $\left.d_{6}\right): \delta=2.43\left(3 \mathrm{H}, \mathrm{s},-\mathrm{CH}_{3}\right), 3.71$ $\left(3 \mathrm{H}, \mathrm{s},-\mathrm{CH}_{3}\right), 4.94\left(2 \mathrm{H}, \mathrm{s},-\mathrm{CH}_{2}-\right), 7.05(1 \mathrm{H}, \mathrm{d}, J=8.13 \mathrm{~Hz}$, Benzimidazole C-H), 7.37-7.43 (3H, m, 4-Fluorophenyl C-H, Benzimidazole C-H), $7.51(1 \mathrm{H}, \mathrm{d}, J=8.13 \mathrm{~Hz}$, Benzimidazole $\mathrm{C}-\mathrm{H}), 7.88(2 \mathrm{H}, \mathrm{d}, J=8.31 \mathrm{~Hz}, 1,4$-disubstituted benzene C-H), 8.11-8.17 (2H, m, 4-Fluorophenyl C-H), $8.32(2 \mathrm{H}$, d, $J=8.31 \mathrm{~Hz}, 1,4$-disubstituted benzene $\mathrm{C}-\mathrm{H}), 12.11(1 \mathrm{H}$, s, Benzimidazole -NH). ${ }^{13} \mathrm{C}-\mathrm{NMR}\left(75 \mathrm{MHz}\right.$, DMSO- $\left.d_{6}\right)$ : $\delta$ (ppm): 21.81, 32.48, 41.10, $116.36(\mathrm{~d}, J=21.8 \mathrm{~Hz}), 124.42$, 127.14, 128.29, 129.19, 130.04, 131.38, 131.51, 131.85, 132.02 (d, J $=9.0 \mathrm{~Hz}), 132.22,132.49,132.51(\mathrm{~d}, J=3.0 \mathrm{~Hz}), 150.44,150.99$, $155.33,165.79(\mathrm{~d}, J=251.3 \mathrm{~Hz}), 192.53 .[\mathrm{M}+\mathrm{H}]^{+}$calcd for $\mathrm{C}_{25} \mathrm{H}_{20} \mathrm{FN}_{5}$ OS: 458.1445 ; found: 458.1443 .

2.1.9. 2-(4-(4-Methyl-5-(2-(4-bromophenyl)-2-oxo-ethylthio)4H-1,2,4-triazol-3yl)-phenyl)-5(6)-methyl-1H-benzimidazole (5d). Yield: $75 \%$. M.p. $259-260^{\circ}$ C. FTIR (ATR, $\mathrm{cm}^{-1}$ ): 3201 $(\mathrm{N}-\mathrm{H}), 1680(\mathrm{C}=\mathrm{O}), 850$ (1,4-disubstituted benzene $\mathrm{C}-\mathrm{H})$. ${ }^{1} \mathrm{H}-\mathrm{NMR}\left(300 \mathrm{MHz}, \mathrm{DMSO}-d_{6}\right): \delta=2.43\left(3 \mathrm{H}, \mathrm{s},-\mathrm{CH}_{3}\right), 3.71$ $\left(3 \mathrm{H}, \mathrm{s},-\mathrm{CH}_{3}\right), 4.92\left(2 \mathrm{H}, \mathrm{s},-\mathrm{CH}_{2}-\right), 7.05(1 \mathrm{H}, \mathrm{s}$, Benzimidazole C-H), 7.34-7.56 (2H, m, Ar-H), 7.78-7.95 (6H, m, Ar-H), 8.31 $(2 \mathrm{H}, \mathrm{s}, \mathrm{Ar}-\mathrm{H}), 12.91(1 \mathrm{H}, \mathrm{s}$, Benzimidazole $-\mathrm{NH}) .{ }^{13} \mathrm{C}-\mathrm{NMR}$ $\left(75 \mathrm{MHz}, \mathrm{DMSO}-d_{6}\right): \delta(\mathrm{ppm}): 21.81,32.48,41.10,111.63$, $119.13,123.99,124.85,127.08,128.39,129.20,130.92,131.33$, $131.96,132.36,132.78,134.77,142.46,150.31,150.90,155.35$, 193.23. $[\mathrm{M}+\mathrm{H}]^{+}$calcd for $\mathrm{C}_{25} \mathrm{H}_{20} \mathrm{BrN}_{5} \mathrm{OS}$ : 518.0645 ; found: 518.0635 .

2.1.10. 2-(4-(4-Methyl-5-(2-(4-methylphenyl)-2-oxo-ethylthio)4H-1,2,4-triazol-3yl)-phenyl)-5(6)-methyl-1H-benzimidazole (5e). Yield: $78 \%$. M.p. $261-262^{\circ}$ C. FTIR (ATR, $\mathrm{cm}^{-1}$ ): 3197 $(\mathrm{N}-\mathrm{H}), 1674(\mathrm{C}=\mathrm{O}), 850$ (1,4-disubstituted benzene $\mathrm{C}-\mathrm{H})$. ${ }^{1} \mathrm{H}-\mathrm{NMR}\left(300 \mathrm{MHz}, \mathrm{DMSO}-d_{6}\right): \delta=2.39\left(3 \mathrm{H}, \mathrm{s},-\mathrm{CH}_{3}\right)$, $2.44\left(3 \mathrm{H}, \mathrm{s},-\mathrm{CH}_{3}\right), 3.70\left(3 \mathrm{H}, \mathrm{s},-\mathrm{CH}_{3}\right), 4.91\left(2 \mathrm{H}, \mathrm{s},-\mathrm{CH}_{2}-\right)$, $7.07(1 \mathrm{H}, \mathrm{d}, J=8.13 \mathrm{~Hz}$, Benzimidazole C-H), $7.37(2 \mathrm{H}, \mathrm{d}, J$ $=8.12$, 4-methylphenyl C-H), $7.41(1 \mathrm{H}$, br.s, Benzimidazole C-H), $7.51(1 \mathrm{H}$, br.s, Benzimidazole C-H $), 7.88(2 \mathrm{H}, \mathrm{d}$,
$J=8.37 \mathrm{~Hz}, 1,4$-disubstituted benzene $\mathrm{C}-\mathrm{H}), 7.93(2 \mathrm{H}, \mathrm{d}$, $J=8.12$, 4-methylphenyl C-H $), 8.31(2 \mathrm{H}, \mathrm{d}, J=8.37 \mathrm{~Hz}$, 1,4-disubstituted benzene C-H), $12.92(1 \mathrm{H}, \mathrm{s}$, Benzimidazole -NH). ${ }^{13} \mathrm{C}-\mathrm{NMR}\left(75 \mathrm{MHz}, \mathrm{DMSO}-d_{6}\right): \delta$ (ppm): 21.68, $21.82,32.48,41.25,111.75,119.21,124.28,127.09,128.28,129.05$, $129.20,129.84,130.77,131.94,132.80,133.22,135.86,144.79$, 150.47, 151.06, 155.31, 193.39. $[\mathrm{M}+\mathrm{H}]^{+}$calcd for $\mathrm{C}_{26} \mathrm{H}_{23} \mathrm{~N}_{5} \mathrm{OS}$ : 454.1696; found: 454.1683 .

2.1.11. 2-(4-(4-Methyl-5-(2-(4-chlorophenyl)-2-oxo-ethylthio)4H-1,2,4-triazol-3yl)-phenyl)-5(6)-methyl-1H-benzimidazole (5f). Yield: $76 \%$. M.p. $226-227^{\circ} \mathrm{C}$. FTIR (ATR, $\mathrm{cm}^{-1}$ ): 3190 $(\mathrm{N}-\mathrm{H}), 1680(\mathrm{C}=\mathrm{O}), 850$ (1,4-disubstituted benzene $\mathrm{C}-\mathrm{H})$. ${ }^{1} \mathrm{H}-\mathrm{NMR}\left(300 \mathrm{MHz}\right.$, DMSO- $\left.d_{6}\right): \delta=2.44\left(3 \mathrm{H}, \mathrm{s},-\mathrm{CH}_{3}\right), 3.71$ $\left(3 \mathrm{H}, \mathrm{s},-\mathrm{CH}_{3}\right), 4.93\left(2 \mathrm{H}, \mathrm{s},-\mathrm{CH}_{2}-\right), 7.04(1 \mathrm{H}, \mathrm{d}, J=8.55 \mathrm{~Hz}$, Benzimidazole C-H), 7.45 ( $1 \mathrm{H}$, br.s, Benzimidazole C-H), 7.57 (1H, br.s, Benzimidazole C-H), $7.64(2 \mathrm{H}, \mathrm{d}, J=8.55,4-$ chlorophenyl C-H), $7.88(2 \mathrm{H}, \mathrm{d}, J=8.33 \mathrm{~Hz}, 1,4$-disubstituted benzene $\mathrm{C}-\mathrm{H}), 8.05(2 \mathrm{H}, \mathrm{d}, \mathrm{J}=8.55$, 4-chlorophenyl C-H), $8.31(2 \mathrm{H}, \mathrm{d}, J=8.33 \mathrm{~Hz}, 1,4$-disubstituted benzene $\mathrm{C}-\mathrm{H})$, $12.93\left(1 \mathrm{H}\right.$, s, Benzimidazole -NH). ${ }^{13} \mathrm{C}-\mathrm{NMR}(75 \mathrm{MHz}$, DMSO- $d_{6}$ ): $\delta$ (ppm): 21.86, 32.49, 41.12, 111.62, 119.12, 123.99, $124.85,127.07,127.13,129.20,129.41,130.86,131.97,132.78$, $134.45,139.15,142.45,144.67,150.90,155.35,193.12 .[\mathrm{M}+\mathrm{H}]^{+}$ calcd for $\mathrm{C}_{25} \mathrm{H}_{20} \mathrm{ClN}_{5} \mathrm{OS}$ : 474.1150; found: 474.1149.

2.1.12. 2-(4-(4-Methyl-5-(2-(2,4-dichlorophenyl)-2-oxo-ethylthio)-4H-1,2,4-triazol-3yl)-phenyl)-5(6)-methyl-1H-benzimidazole (5g). Yield: $74 \%$. M.p. $159-160^{\circ}$ C. FTIR (ATR, $\mathrm{cm}^{-1}$ ): $3305(\mathrm{~N}-\mathrm{H}), 1685(\mathrm{C}=\mathrm{O}), 854$ (1,4-disubstituted benzene CH). ${ }^{1} \mathrm{H}-\mathrm{NMR}\left(300 \mathrm{MHz}, \mathrm{DMSO}-d_{6}\right): \delta=2.45\left(3 \mathrm{H}, \mathrm{s},-\mathrm{CH}_{3}\right)$, $3.68\left(3 \mathrm{H}, \mathrm{s},-\mathrm{CH}_{3}\right), 4.81\left(2 \mathrm{H}, \mathrm{s},-\mathrm{CH}_{2}-\right), 7.10(1 \mathrm{H}, \mathrm{d}, J=$ $8.22 \mathrm{~Hz}$, Benzimidazole C-H), $7.43(1 \mathrm{H}, \mathrm{s}$, Benzimidazole C-H), $7.54(1 \mathrm{H}, \mathrm{d}, J=7.98 \mathrm{~Hz}$, Benzimidazole C-H), $7.61(1 \mathrm{H}$, dd, $J=8.38 \mathrm{~Hz}-2.01 \mathrm{~Hz}$ Dichlorophenyl C-H), $7.78(1 \mathrm{H}, \mathrm{d}$, $J=1.98 \mathrm{~Hz}$, Dichloropheyl), $7.90(3 \mathrm{H}, \mathrm{m}, 1,4$-disubstituted benzene C-H, Dichlorophenyl C-H), $8.33(2 \mathrm{H}, \mathrm{d}, J=8.43 \mathrm{~Hz}$, 1,4-disubstituted benzene C-H), $13.28(1 \mathrm{H}, \mathrm{s}$, Benzimidazole -NH). ${ }^{13} \mathrm{C}-\mathrm{NMR}\left(75 \mathrm{MHz}\right.$, DMSO- $\left.d_{6}\right): \delta$ (ppm): 21.81, 32.09, $43.05,111.51,119.19,124.00,124.88,127.24,128.05,128.32,129.25$, $130.56,131.98,132.07,132.11,133.41,134.14,135.82,137.27,143.97$, 150.22, 154.89, 194.94. $[\mathrm{M}+\mathrm{H}]^{+}$calcd for $\mathrm{C}_{25} \mathrm{H}_{19} \mathrm{Cl}_{2} \mathrm{~N}_{5} \mathrm{OS}$ : 508.0760; found: 508.0755.

2.1.13. 2-(4-(4-Methyl-5-(2-(2,4-difluorophenyl)-2-oxo-ethylthio)-4H-1,2,4-triazol-3yl)-phenyl)-5(6)-methyl-1H-benzimidazole (5h). Yield: $82 \%$. M.p. $258-259^{\circ}$ C. FTIR (ATR, $\mathrm{cm}^{-1}$ ): $3196(\mathrm{~N}-\mathrm{H}), 1676(\mathrm{C}=\mathrm{O}), 850$ (1,4-disubstituted benzene CH). ${ }^{1} \mathrm{H}-\mathrm{NMR}\left(300 \mathrm{MHz}, \mathrm{DMSO}-d_{6}\right): \delta=2.44\left(3 \mathrm{H}, \mathrm{s},-\mathrm{CH}_{3}\right)$, $3.71\left(3 \mathrm{H}, \mathrm{s},-\mathrm{CH}_{3}\right), 4.83\left(2 \mathrm{H}, \mathrm{s},-\mathrm{CH}_{2}-\right), 7.05(1 \mathrm{H}, \mathrm{d}, J=8.40 \mathrm{~Hz}$, Benzimidazole C-H), 7.25-7.34 (2H, m, Ar-H), 7.43-7.58 (2H, $\mathrm{m}, \mathrm{Ar}-\mathrm{H}), 7.89(2 \mathrm{H}, \mathrm{d}, J=8.33 \mathrm{~Hz}, 1,4$-disubstituted benzene C-H), $7.98-8.06(1 \mathrm{H}, \mathrm{m}, \mathrm{Ar}-\mathrm{H}), 8.31(2 \mathrm{H}, \mathrm{d}, J=8.33 \mathrm{~Hz}$, 1,4-disubstituted benzene $\mathrm{C}-\mathrm{H}), 12.92(1 \mathrm{H}, \mathrm{s}$, Benzimidazole -NH). ${ }^{13} \mathrm{C}-\mathrm{NMR}\left(75 \mathrm{MHz}, \mathrm{DMSO}-d_{6}\right): \delta(\mathrm{ppm}): 21.86$, $32.45,44.29,105.79(\mathrm{t}, J=26.81 \mathrm{~Hz}), 111.62,113.03(\mathrm{dd}, J=$ $24.98 \mathrm{~Hz}-3.31 \mathrm{~Hz}), 119.12,123.99,124.85,127.07,128.22,129.21$, $131.96,132.77,133.38(\mathrm{dd}, J=10.97 \mathrm{~Hz}-3.83 \mathrm{~Hz}), 135.80,142.45$, 
144.67, 150.94, 155.34, 164.0, 164.24, 190.38 (d, $J=3.76 \mathrm{~Hz}$ ). $[\mathrm{M}+\mathrm{H}]^{+}$calcd for $\mathrm{C}_{25} \mathrm{H}_{19} \mathrm{~F}_{2} \mathrm{~N}_{5}$ OS: 476.1351; found: 476.1348 .

2.1.14. 2-(4-(4-Methyl-5-(2-(3,4-dihydroxyphenyl)-2-oxo-ethylthio)-4H-1,2,4-triazol-3yl)-phenyl)-5(6)-methyl-1H-benzimidazole (5i). Yield: 79\%. M.p. 249-250 C. FTIR (ATR, $\left.\mathrm{cm}^{-1}\right)$ : 3041 (N-H), $1681(\mathrm{C}=\mathrm{O}), 854$ (1,4-disubstituted benzene CH). ${ }^{1} \mathrm{H}-\mathrm{NMR}\left(300 \mathrm{MHz}, \mathrm{DMSO}-d_{6}\right): \delta=2.43\left(3 \mathrm{H}, \mathrm{s},-\mathrm{CH}_{3}\right)$, $3.70\left(3 \mathrm{H}, \mathrm{s},-\mathrm{CH}_{3}\right), 4.81\left(2 \mathrm{H}, \mathrm{s},-\mathrm{CH}_{2}-\right), 6.85(1 \mathrm{H}, \mathrm{d}, J=$ $8.25 \mathrm{~Hz}$, Dihydroxyphenyl C-H), $7.05(1 \mathrm{H}, \mathrm{d}, J=7.95 \mathrm{~Hz}$, Benzimidazole C-H), 7.39-7.46 (4H, m, Dihydroxyphenyl C-H, Benzimidazole C-H), $7.88(2 \mathrm{H}, \mathrm{d}, \mathrm{J}=8.45 \mathrm{~Hz}, 1,4-$ disubstituted benzene $\mathrm{C}-\mathrm{H}), 8.31(2 \mathrm{H}, \mathrm{d}, J=8.45 \mathrm{~Hz}, 1,4-$ disubstituted benzene $\mathrm{C}-\mathrm{H}), 12.86(1 \mathrm{H}, \mathrm{s}$, Benzimidazole $\mathrm{NH}) .{ }^{13} \mathrm{C}-\mathrm{NMR}\left(75 \mathrm{MHz}, \mathrm{DMSO}-d_{6}\right): \delta$ (ppm): 21.81, 32.45, 41.07, 111.53, 115.63, 119.09, 119.25, 122.52, 126.26, 127.09, 127.47, $128.31,129.04,129.20,130.44,131.92,134.68,138.29,145.85$, 151.26, 151.91, 155.28, 191.88. $[\mathrm{M}+\mathrm{H}]^{+}$calcd for $\mathrm{C}_{25} \mathrm{H}_{21} \mathrm{~N}_{5} \mathrm{O}_{3} \mathrm{~S}$ : 472.1438; found: 472.1439 .

2.1.15. 2-(4-(4-Ethyl-5-(2-phenyl-2-oxo-ethylthio)-4H-1,2,4-triazol-3yl)-phenyl)-5(6)-methyl-1H-benzimidazole (5j). Yield: 83\%. M.p. $235-236^{\circ}$ C. FTIR (ATR, $\mathrm{cm}^{-1}$ ): $3255(\mathrm{~N}-\mathrm{H})$, $1680(\mathrm{C}=\mathrm{O}), 848$ (1,4-disubstituted benzene $\mathrm{C}-\mathrm{H}) .{ }^{1} \mathrm{H}-\mathrm{NMR}$ $\left(300 \mathrm{MHz}, \mathrm{DMSO}-d_{6}\right): \delta=1.29\left(3 \mathrm{H}, \mathrm{t}, J=7.14,-\mathrm{CH}_{3}\right)$, $2.50\left(3 \mathrm{H}, \mathrm{s},-\mathrm{CH}_{3}\right), 4.15\left(2 \mathrm{H}, \mathrm{q}, J=7.14 \mathrm{~Hz},-\mathrm{CH}_{2}\right), 5.04$ $\left(2 \mathrm{H}, \mathrm{s},-\mathrm{CH}_{2}^{-}\right), 7.33(1 \mathrm{H}, \mathrm{d}, J=8.31 \mathrm{~Hz}$, Benzimidazole C-H), 7.55-7.60 (3H, m, Benzimidazole C-H, Monosubstituted Benzene C-H), 7.69-7.72 (2H, m, Benzimidazole C-H, Monosubstituted Benzene C-H), $7.99(2 \mathrm{H}, \mathrm{d}, J=8.24 \mathrm{~Hz}$, 1,4-disubstituted benzene C-H), $8.06(2 \mathrm{H}, \mathrm{d}, J=7.44 \mathrm{~Hz}$, Monosubstituted Benzene C-H), $8.39(2 \mathrm{H}, \mathrm{d}, J=8.24 \mathrm{~Hz}$, 1,4-disubstituted benzene C-H), $13.24(1 \mathrm{H}, \mathrm{s}$, Benzimidazole -NH). ${ }^{13} \mathrm{C}-\mathrm{NMR}$ (75 MHz, DMSO-d6): $\delta$ (ppm): 15.49, 21.72, 40.80, 41.21, 114.19, 114.65, 126.88, 127.21, 128.52, 128.91, $129.33,129.57,130.75,132.69,134.28,134.52,135.59,135.76$, 148.69, 151.09, 154.27, 193.63. $[\mathrm{M}+\mathrm{H}]^{+}$calcd for $\mathrm{C}_{26} \mathrm{H}_{23} \mathrm{~N}_{5} \mathrm{OS}$ : 454.1696; found: 454.1683 .

2.1.16. 2-(4-(4-Ethyl-5-(2-(4-cyanophenyl)-2-oxo-ethylthio)4H-1,2,4-triazol-3yl)-phenyl)-5(6)-methyl-1H-benzimidazole (5k). Yield: $80 \%$. M.p. $181-182^{\circ}$ C. FTIR (ATR, $\mathrm{cm}^{-1}$ ): 2987 $(\mathrm{N}-\mathrm{H}), 1676(\mathrm{C}=\mathrm{O}), 849$ (1,4-disubstituted benzene $\mathrm{C}-\mathrm{H})$. ${ }^{1} \mathrm{H}-\mathrm{NMR}\left(300 \mathrm{MHz}, \mathrm{DMSO}-d_{6}\right): \delta=1.28(3 \mathrm{H}, \mathrm{t}, J=7.20$, $\left.-\mathrm{CH}_{3}\right), 2.44\left(3 \mathrm{H}, \mathrm{s},-\mathrm{CH}_{3}\right), 4.12\left(2 \mathrm{H}, \mathrm{q}, J=7.16 \mathrm{~Hz},-\mathrm{CH}_{2}\right)$, $5.04\left(2 \mathrm{H}, \mathrm{s},-\mathrm{CH}_{2}-\right), 7.06(1 \mathrm{H}, \mathrm{d}, J=8.28 \mathrm{~Hz}$, Benzimidazole C-H), $7.41(1 \mathrm{H}, \mathrm{s}$, Benzimidazole $\mathrm{C}-\mathrm{H}), 7.52(1 \mathrm{H}, \mathrm{d}, J=$ $8.28 \mathrm{~Hz}$, Benzimidazole C-H), $7.82(2 \mathrm{H}, \mathrm{d}, J=8.49 \mathrm{~Hz}$, 1,4-disubstituted benzene C-H), $8.07(2 \mathrm{H}, \mathrm{d}, J=8.55$, 4-cyanophenyl C-H), $8.20(2 \mathrm{H}, \mathrm{d}, J=8.55,4$-cyanophenyl C-H), $8.31(2 \mathrm{H}, \mathrm{d}, J=8.46 \mathrm{~Hz}, 1,4$-disubstituted benzene $\mathrm{C}-\mathrm{H}), 12.96$ (1H, s, Benzimidazole -NH). ${ }^{13} \mathrm{C}-\mathrm{NMR}(75 \mathrm{MHz}$, DMSO- $\left.d_{6}\right): \delta$ (ppm): 15.52, 21.83, 41.13, 116.03, 118.57, 121.93, $123.66,127.25,128.42,129.25,129.54,131.74,132.03,133.34$, 134.03, 137.00, 139.10, 142.84, 150.28, 150.43, 154.86, 193.40. $[\mathrm{M}+\mathrm{H}]^{+}$calcd for $\mathrm{C}_{27} \mathrm{H}_{22} \mathrm{~N}_{6} \mathrm{OS}: 479.1649$; found: 479.1640 .

2.1.17. 2-(4-(4-Ethyl-5-(2-(4-fluorophenyl)-2-oxo-ethylthio)4H-1,2,4-triazol-3yl)-phenyl)-5(6)-methyl-1H-benzimidazole (5l). Yield: $78 \%$. M.p. $241-242^{\circ}$ C. FTIR (ATR, $\mathrm{cm}^{-1}$ ): 3142
(N-H), $1683(\mathrm{C}=\mathrm{O}), 850$ (1,4-disubstituted benzene C-H). ${ }^{1} \mathrm{H}-\mathrm{NMR}\left(300 \mathrm{MHz}, \mathrm{DMSO}-d_{6}\right): \delta=1.28(3 \mathrm{H}, \mathrm{t}, J=7.13$, $\left.-\mathrm{CH}_{3}\right), 2.43\left(3 \mathrm{H}, \mathrm{s},-\mathrm{CH}_{3}\right), 4.12\left(2 \mathrm{H}, \mathrm{q}, J=7.13 \mathrm{~Hz},-\mathrm{CH}_{2}\right), 5.00$ $\left(2 \mathrm{H}, \mathrm{s},-\mathrm{CH}_{2}-\right), 7.05(1 \mathrm{H}, \mathrm{d}, J=8.28 \mathrm{~Hz}$, Benzimidazole C-H), 7.38-7.43 (3H, m, Fluorophenyl C-H, Benzimidazole C-H), $7.56(1 \mathrm{H}, \mathrm{d}, J=8.28 \mathrm{~Hz}$, Benzimidazole C-H), $7.82(2 \mathrm{H}, \mathrm{d}, J$ $=8.16 \mathrm{~Hz}, 1,4$-disubstituted benzene $\mathrm{C}-\mathrm{H}), 8.12-8.17(2 \mathrm{H}, \mathrm{m}$, Fluorophenyl C-H), 8.33 (2H, d, $J=8.16 \mathrm{~Hz}, 1,4$-disubstituted benzene C-H), 13.05 (1H, s, Benzimidazole -NH). ${ }^{13} \mathrm{C}-\mathrm{NMR}$ $\left(75 \mathrm{MHz}, \mathrm{DMSO}-d_{6}\right): \delta$ (ppm): 15.53, 21.80, 40.80, 41.11, $116.37(\mathrm{~d}, J=21.9 \mathrm{~Hz}), 119.10,119.22,123.96,124.82,128.37$, 129.22, 131.29, 132.02 (d, $J=9.5 \mathrm{~Hz}), 132.55$ (d, $J=3.0 \mathrm{~Hz})$, $135.85,142.44,144.65,150.28,150.46,154.83,165.78(\mathrm{~d}, J=$ 251.1 Hz), 192.41. $[\mathrm{M}+\mathrm{H}]^{+}$calcd for $\mathrm{C}_{26} \mathrm{H}_{22} \mathrm{FN}_{5} \mathrm{OS}$ : 472.1602; found: 472.1604 .

2.1.18. 2-(4-(4-Ethyl-5-(2-(4-bromophenyl)-2-oxo-ethylthio)4H-1,2,4-triazol-3yl)-phenyl)-5(6)-methyl-1H-benzimidazole (5m). Yield: $81 \%$. M.p. $186-187^{\circ}$ C. FTIR (ATR, $\mathrm{cm}^{-1}$ ): 3122 (N-H), 1680 (C=O), 850 (1,4-disubstituted benzen). ${ }^{1} \mathrm{H}$ $\operatorname{NMR}\left(300 \mathrm{MHz}, \mathrm{DMSO}-d_{6}\right): \delta=1.28\left(3 \mathrm{H}, \mathrm{t}, J=7.16,-\mathrm{CH}_{3}\right)$, $2.44\left(3 \mathrm{H}, \mathrm{s},-\mathrm{CH}_{3}\right), 4.11\left(2 \mathrm{H}, \mathrm{q}, J=7.16 \mathrm{~Hz},-\mathrm{CH}_{2}\right), 4.99(2 \mathrm{H}$, $\left.\mathrm{s},-\mathrm{CH}_{2}-\right), 7.06(\mathrm{H}, \mathrm{d}, J=8.18 \mathrm{~Hz}$, Benzimidazole $\mathrm{C}-\mathrm{H})$, $7.41(1 \mathrm{H}, \mathrm{s}$, Benzimidazole C-H), $7.52(1 \mathrm{H}, \mathrm{d}, J=8.18 \mathrm{~Hz}$, Benzimidazole C-H), 7.78-7.84 (4H, m, 1,4-disubstituted benzene, 4-bromophenyl), $7.98(2 \mathrm{H}, \mathrm{d}, J=8.55 \mathrm{~Hz}, 4$ bromophenyl), $8.32(2 \mathrm{H}, \mathrm{d}, J=8.37 \mathrm{~Hz}, 1,4$-disubstituted benzene C-H), 12.97 (1H, s, Benzimidazole -NH). ${ }^{13} \mathrm{C}$ NMR $\left(75 \mathrm{MHz}, \mathrm{DMSO}-d_{6}\right): \delta$ (ppm): 15.53, 21.81, 40.79, $41.06,112.35,120.74,124.48,127.05,127.28,128.39,128.49$, $128.74,129.26,130.92,131.44,131.89,132.38,133.18,134.81$, $140.65,150.38,150.41,154.81,193.10 .[\mathrm{M}+\mathrm{H}]^{+}$calcd for $\mathrm{C}_{26} \mathrm{H}_{22} \mathrm{BrN}_{5} \mathrm{OS}$ : 532.0801; found: 532.0801.

2.1.19. 2-(4-(4-Ethyl-5-(2-(4-methylphenyl)-2-oxo-ethylthio)4H-1,2,4-triazol-3yl)-phenyl)-5(6)-methyl-1H-benzimidazole (5n). Yield: 76\%. M.p. 249-250 C. FTIR (ATR, $\mathrm{cm}^{-1}$ ): $3163(\mathrm{~N}-\mathrm{H}), 1678$ (C=O), 852 (1,4-disubstituted benzen). ${ }^{1} \mathrm{H}-\mathrm{NMR}\left(300 \mathrm{MHz}, \mathrm{DMSO}-d_{6}\right): \delta=1.27(3 \mathrm{H}, \mathrm{t}, J=7.19$, $\left.-\mathrm{CH}_{3}\right), 2.39\left(3 \mathrm{H}, \mathrm{s},-\mathrm{CH}_{3}\right), 2.43\left(3 \mathrm{H}, \mathrm{s},-\mathrm{CH}_{3}\right), 4.11(2 \mathrm{H}$, $\left.\mathrm{q}, J=7.17 \mathrm{~Hz},-\mathrm{CH}_{2}\right), 4.98\left(2 \mathrm{H}, \mathrm{s},-\mathrm{CH}_{2}-\right), 7.06(1 \mathrm{H}, \mathrm{d}$, $J=8.22 \mathrm{~Hz}$, Benzimidazole $\mathrm{C}-\mathrm{H}), 7.37(2 \mathrm{H}, \mathrm{d}, J=8.09$, 4-methylphenyl C-H), $7.41(1 \mathrm{H}, \mathrm{s}$, Benzimidazole $\mathrm{C}-\mathrm{H})$, $7.52(1 \mathrm{H}, \mathrm{d}, J=8.22 \mathrm{~Hz}$, Benzimidazole C-H), $7.82(2 \mathrm{H}, \mathrm{d}$, $J=8.37 \mathrm{~Hz}, 1,4$-disubstituted benzene $\mathrm{C}-\mathrm{H}), 7.94(2 \mathrm{H}, \mathrm{d}$, $J=8.09$, 4-methylphenyl C-H $), 8.32(2 \mathrm{H}, \mathrm{d}, J=8.37 \mathrm{~Hz}$, 1,4-disubstituted benzene $\mathrm{C}-\mathrm{H}), 12.71(1 \mathrm{H}, \mathrm{s}$, Benzimidazole -NH) ${ }^{13} \mathrm{C}-\mathrm{NMR}\left(75 \mathrm{MHz}, \mathrm{DMSO}-d_{6}\right): \delta(\mathrm{ppm}): 15.53,21.68$, 21.81, 36.24, 41.22, 114.96, 115.51, 115.96, 124.43, 127.26, 128.50, $129.03,129.24,129.84,130.84,131.95,132.24,133.26,144.78$, 150.42, 150.58, 154.78, 193.21. $[\mathrm{M}+\mathrm{H}]^{+}$calcd for $\mathrm{C}_{27} \mathrm{H}_{25} \mathrm{~N}_{5} \mathrm{OS}$ : 468.1853; found: 468.1856 .

2.1.20. 2-(4-(4-Ethyl-5-(2-(4-chlorophenyl)-2-oxo-ethylthio)4H-1,2,4-triazol-3yl)-phenyl)-5(6)-methyl-1H-benzimidazole (5o). Yield: $79 \%$. M.p. $237-238^{\circ}$ C. FTIR (ATR, $\mathrm{cm}^{-1}$ ): 3045 $(\mathrm{N}-\mathrm{H}), 1695(\mathrm{C}=\mathrm{O}), 847$ (1,4-disubstituted benzene $\mathrm{C}-\mathrm{H})$. ${ }^{1} \mathrm{H}-\mathrm{NMR}\left(300 \mathrm{MHz}, \mathrm{DMSO}-d_{6}\right): \delta=1.27(3 \mathrm{H}, \mathrm{t}, J=7.10$, 
$\left.-\mathrm{CH}_{3}\right), 2.44\left(3 \mathrm{H}, \mathrm{s},-\mathrm{CH}_{3}\right), 4.11\left(2 \mathrm{H}\right.$, br.s, $\left.-\mathrm{CH}_{2}\right), 5.00(2 \mathrm{H}$, s, $\left.-\mathrm{CH}_{2}-\right), 7.06(1 \mathrm{H}, \mathrm{d}, J=8.01 \mathrm{~Hz}$, Benzimidazole $\mathrm{C}-\mathrm{H})$, $7.41(1 \mathrm{H}, \mathrm{s}$, Benzimidazole C-H), $7.51(1 \mathrm{H}, \mathrm{d}, J=6.87 \mathrm{~Hz}$, Benzimidazole C-H), 7.65 ( $2 \mathrm{H}, \mathrm{d}, J=8.62$, 4-chlorophenyl $\mathrm{C}-\mathrm{H}), 7.82(2 \mathrm{H}, \mathrm{d}, J=8.35 \mathrm{~Hz}, 1,4$-disubstituted benzene $\mathrm{C}-\mathrm{H}), 8.06(2 \mathrm{H}, \mathrm{d}, J=8.62$, 4-chlorophenyl C-H), $8.31(2 \mathrm{H}$, d, $J=8.35 \mathrm{~Hz}, 1,4$-disubstituted benzene $\mathrm{C}-\mathrm{H}), 12.93(1 \mathrm{H}$, s, Benzimidazole -NH). ${ }^{13} \mathrm{C}-\mathrm{NMR}\left(75 \mathrm{MHz}, \mathrm{DMSO}-d_{6}\right): \delta$ (ppm): 15.52, 21.81, 40.80, 41.08, 115.03, 124.41, 124.60, 127.24, $128.43,129.25,129.43,130.85,131.10,132.03,132.27,134.50$, $139.16,142.37,150.42,153.03,154.83,192.88 .[\mathrm{M}+\mathrm{H}]^{+}$calcd for $\mathrm{C}_{26} \mathrm{H}_{22} \mathrm{ClN}_{5} \mathrm{OS}$ : 488.1306; found: 488.1304 .

2.1.21. 2-(4-(4-Ethyl-5-(2-(2,4-dichlorophenyl)-2-oxo-ethylthio)-4H-1,2,4-triazol-3yl)-phenyl)-5(6)-methyl-1H-benzimidazole (5p). Yield: $75 \%$. M.p. $211-212^{\circ} \mathrm{C}$. FTIR $\left(\mathrm{ATR}, \mathrm{cm}^{-1}\right.$ ): $2920(\mathrm{~N}-\mathrm{H}), 1670(\mathrm{C}=\mathrm{O}), 864$ (1,4-disubstituted benzene C-H). ${ }^{1} \mathrm{H}-\mathrm{NMR}\left(300 \mathrm{MHz}, \mathrm{DMSO}-d_{6}\right): \delta=1.25(3 \mathrm{H}, \mathrm{t}$, $J=7.19,-\mathrm{CH} 3), 2.44\left(3 \mathrm{H}, \mathrm{s},-\mathrm{CH}_{3}\right), 4.07(2 \mathrm{H}, \mathrm{q}, J=$ $\left.7.10 \mathrm{~Hz},-\mathrm{CH}_{2}\right), 4.85\left(2 \mathrm{H}, \mathrm{s},-\mathrm{CH}_{2}-\right), 7.04(1 \mathrm{H}, \mathrm{d}, J=8.16 \mathrm{~Hz}$, Benzimidazole C-H), 7.40 ( $2 \mathrm{H}$, br.s, Benzimidazole C-H), $7.60(1 \mathrm{H}, \mathrm{dd}, J=8.37 \mathrm{~Hz}-1.98 \mathrm{~Hz}$ Dichlorophenyl C-H $)$, $7.76(1 \mathrm{H}, \mathrm{d}, J=1.92 \mathrm{~Hz}$, Dichlorophenyl C-H $), 7.82(2 \mathrm{H}$, $\mathrm{d}, J=8.36 \mathrm{~Hz}, 1,4$-disubstituted benzene $\mathrm{C}-\mathrm{H}), 7.89(1 \mathrm{H}$, d, $J=8.40 \mathrm{~Hz}$, Dichlorophenyl C-H $), 8.32(2 \mathrm{H}, \mathrm{d}, J=$ $8.36 \mathrm{~Hz}, 1,4$-disubstituted benzene C-H), $12.91(1 \mathrm{H}, \mathrm{s}$, Benzimidazole -NH). ${ }^{13} \mathrm{C}-\mathrm{NMR}\left(75 \mathrm{MHz}, \mathrm{DMSO}-d_{6}\right): \delta(\mathrm{ppm})$ : 15.46, 21.81, 43.05, 111.51, 119.19, 120.61, 122.55, 124.00, 127.24, $128.05,128.32,129.25,130.56,131.98,132.07,132.11,132.80$, $135.82,137.27,142.48,150.22,154.89,194.94 .[\mathrm{M}+\mathrm{H}]^{+}$calcd for $\mathrm{C}_{26} \mathrm{H}_{21} \mathrm{Cl}_{2}$ NOS: 523.0917; found: 523.0912.

2.1.22. 2-(4-(4-Ethyl-5-(2-(2,4-difluorophenyl)-2-oxo-ethylthio)-4H-1,2,4-triazol-3yl)-phenyl)-5(6)-methyl-1H-benzimidazole (5o). Yield: $73 \%$. M.p. $239-240^{\circ}$ C. FTIR (ATR, $\mathrm{cm}^{-1}$ ): $3045(\mathrm{~N}-\mathrm{H}), 1699(\mathrm{C}=\mathrm{O}), 847$ (1,4-disubstituted benzene CH). ${ }^{1} \mathrm{H}-\mathrm{NMR}\left(300 \mathrm{MHz}, \mathrm{DMSO}-d_{6}\right): \delta=1.28(3 \mathrm{H}, \mathrm{t}, J=7.20$, $\left.-\mathrm{CH}_{3}\right), 2.44\left(3 \mathrm{H}, \mathrm{s},-\mathrm{CH}_{3}\right), 4.11\left(2 \mathrm{H}, \mathrm{q}, J=7.14 \mathrm{~Hz},-\mathrm{CH}_{2}\right), 4.90$ $\left(2 \mathrm{H}, \mathrm{s},-\mathrm{CH}_{2}-\right), 7.06(1 \mathrm{H}, \mathrm{d}, J=7.20 \mathrm{~Hz}$, Benzimidazole C$\mathrm{H}), 7.29(1 \mathrm{H}, \mathrm{td}, J=8.44 \mathrm{~Hz}-2.32 \mathrm{~Hz}$, Difluorophenyl C-H), $7.41(1 \mathrm{H}, \mathrm{s}$, Benzimidazole C- $\mathrm{H}), 7.41-7.54(2 \mathrm{H}, \mathrm{m}$, Benzimidazole C-H, Difluorophenyl C-H), $7.83(2 \mathrm{H}, \mathrm{d}, J=8.42 \mathrm{~Hz}$, 1,4-disubstituted benzene C-H), 7.99-8.07 $(1 \mathrm{H}, \mathrm{m}$, Difluorophenyl C-H $), 8.31(2 \mathrm{H}, \mathrm{d}, J=8.42 \mathrm{~Hz}, 1,4$-disubstituted benzene $\mathrm{C}-\mathrm{H}), 12.96(1 \mathrm{H}, \mathrm{s}$, Benzimidazole $-\mathrm{NH}) .{ }^{13} \mathrm{C}-\mathrm{NMR}$ $\left(75 \mathrm{MHz}, \mathrm{DMSO}-d_{6}\right): \delta(\mathrm{ppm}): 15.50,21.81,44.20,44.30$, $105.81(\mathrm{t}, J=26.8 \mathrm{~Hz}), 113.04(\mathrm{dd}, J=21.6 \mathrm{~Hz}-3.6 \mathrm{~Hz}), 123.38$, $124.40,127.05,127.24,128.42,128.74129 .26,132.02,132.22$, $133.37(\mathrm{dd}, J=11.1 \mathrm{~Hz}-3.6 \mathrm{~Hz}), 135.80,137.99,142.60,150.40$, $154.84,163.09(\mathrm{dd}, J=234.1 \mathrm{~Hz}-11.7 \mathrm{~Hz}), 165.86(\mathrm{dd}, J=$ $255.0 \mathrm{~Hz}-12.8 \mathrm{~Hz}), 190.30(\mathrm{~d}, J=4.1 \mathrm{~Hz}) .[\mathrm{M}+\mathrm{H}]^{+}$calcd for $\mathrm{C}_{26} \mathrm{H}_{21} \mathrm{~F}_{2} \mathrm{~N}_{5} \mathrm{OS}$ : 490.1505; found: 490.1494 .

2.1.23. 2-(4-(4-Ethyl-5-(2-(3,4-dihydroxyphenyl)-2-oxo-ethylthio)-4H-1,2,4-triazol-3yl)-phenyl)-5(6)-methyl-1H-benzimidazole (5s). Yield: $84 \%$. M.p. $252-253^{\circ}$ C. FTIR (ATR, $\mathrm{cm}^{-1}$ ): $3300(\mathrm{~N}-\mathrm{H}), 1683(\mathrm{C}=\mathrm{O}), 852$ (1,4-disubstituted benzen). ${ }^{1} \mathrm{H}-$ $\operatorname{NMR}\left(300 \mathrm{MHz}, \mathrm{DMSO}-d_{6}\right): \delta=1.27\left(3 \mathrm{H}, \mathrm{t}, J=7.17,-\mathrm{CH}_{3}\right)$, $2.44\left(3 \mathrm{H}, \mathrm{s},-\mathrm{CH}_{3}\right), 4.11\left(2 \mathrm{H}, \mathrm{q}, J=7.10 \mathrm{~Hz},-\mathrm{CH}_{2}\right), 4.88(2 \mathrm{H}$, s, $\left.-\mathrm{CH}_{2}-\right), 6.86(1 \mathrm{H}, \mathrm{d}, J=8.25 \mathrm{~Hz}$, Dihydroxyphenyl C-H), $7.05(1 \mathrm{H}, \mathrm{d}, J=7.23 \mathrm{~Hz}$, Benzimidazole C-H), 7.35-7.55 $(4 \mathrm{H}$, $\mathrm{m}$, Dihydroxyphenyl C-H, Benzimidazole C-H), $7.82(2 \mathrm{H}$, d, $J=8.40 \mathrm{~Hz}, 1,4$-disubstituted benzene $\mathrm{C}-\mathrm{H}), 8.31(2 \mathrm{H}$, d, $J=8.40 \mathrm{~Hz}, 1,4$-disubstituted benzene $\mathrm{C}-\mathrm{H}), 9.47(1 \mathrm{H}, \mathrm{s}$, $-\mathrm{OH}), 10.04(1 \mathrm{H}, \mathrm{s},-\mathrm{OH}), 12.92(1 \mathrm{H}, \mathrm{s}$, Benzimidazole $-\mathrm{NH})$.

${ }^{13} \mathrm{C}-\mathrm{NMR}\left(75 \mathrm{MHz}\right.$, DMSO- $\left.d_{6}\right): \delta(\mathrm{ppm}): 15.53,21.81,36.25$, 41.07, 111.53, 115.66, 119.10, 119.27, 122.49, 124.03, 124.82, 127.23, $127.54,128.48,129.25,132.04,132.83,133.57,144.63,145.83$, 150.76, 151.85, 154.76, 191.74. $[\mathrm{M}+\mathrm{H}]^{+}$calcd for $\mathrm{C}_{26} \mathrm{H}_{23} \mathrm{~N}_{5} \mathrm{O}_{3} \mathrm{~S}$ : 486.1594; found: 486.1592 .

2.2. Antifungal Activity. The antifungal activity of synthesized compounds $\mathbf{5 a - 5 s}$ against Candida species was performed according to EUCAST definitive method EDef 7.1 [29]. Briefly, cultures of four fungi (C. glabrata (ATCC 90030), C. krusei (ATCC 6258), C. parapsilosis (ATCC 22019), and C. albicans (ATCC 24433)) were grown in RPMI medium, after an overnight incubation at $37^{\circ} \mathrm{C}$. Fungi suspension was adjusted to approximately $0.5-2.5 \times 10^{5} \mathrm{cfu} / \mathrm{mL}$ with RPMI medium. The test was carried out for medium at $\mathrm{pH}$ $=7$ and twofold dilution was carried out in a 96-well plate to obtain the series dilutions with the concentrations ranging from 0.78 to $800 \mu \mathrm{g} / \mathrm{mL}$. The last well on the microplates, which was containing only the inoculated broth, was kept as control, and the last well with no growth of microorganism was recorded to represent the minimum inhibitory concentration $\left(\mathrm{MIC}_{50}\right)$ in $\mu \mathrm{g} / \mathrm{mL}$. The 96 -well plates were incubated for $24 \mathrm{~h}$, and, at the end of the incubation, resazurin $(20 \mu \mathrm{g} / \mathrm{mL})$ was added to each well to control the growth in the wells. Final plates including microorganism strains were incubated for $2 \mathrm{~h}$. $\mathrm{MIC}_{50}$ values were determined using microplate reader at $590 \mathrm{~nm}$ excitation and $560 \mathrm{~nm}$ emission wavelengths; $\mathrm{MIC}_{50}$ readings were performed twice for entire compounds. Ketoconazole and fluconazole were used as controls.

2.3. Quantification of Ergosterol Level. Total intracellular sterols were extracted as described previously [30]. Sabouraud dextrose broth (50 mL, Difco), enclosing 0, 0.78, 1.56, and $3.125 \mu \mathrm{g} / \mathrm{mL}$ of compounds $\mathbf{5 i}, \mathbf{5 s}$, and positive controls were inoculated with a single C. albicans colony from an overnight Sabouraud dextrose agar plate culture. After incubation for $16 \mathrm{~h}$ at $35^{\circ} \mathrm{C}$, the stationary-phase cells were separated by a centrifugation at 2,700 rpm (Hettich, Rotina $380 \mathrm{R}$, Germany) for $5 \mathrm{~min}$. Isolated cells were washed with sterile distilled water; alcoholic potassium hydroxide solution $(25 \%, 3 \mathrm{~mL})$ was added to each pellet and vortexed for $1 \mathrm{~min}$. Cell suspensions in sterile borosilicate glass tubes were incubated in a water bath $\left(85^{\circ} \mathrm{C}\right)$ for $1 \mathrm{~h}$. After cooling to room temperature, sterols were extracted in the water: chloroform $(1: 3 \mathrm{~mL})$ mixture by a vigorous vortex mixing for $3 \mathrm{~min}$. The chloroform layer was separated and sterol extract $(1 \mu \mathrm{L})$ was injected to LC-MSMS system (Shimadzu LCMS 8040, Kyoto, Japan). The mass spectrometric analysis conditions were provided as reported in our recent study [31]. Level of ergosterol in negative control samples was considered as $100 \%$. All samples, including different concentrations of 
compounds (5i and $\mathbf{5 s}$ ) and reference agents, were analyzed in quadruplicate, and the data were stated as mean \pm standard deviation (SD).

2.4. Cytotoxicity Test. NIH/3T3 mouse embryonic fibroblast cell line (ATCC CRL 1658) was used in the cytotoxicity test. The cells were incubated as reported in the supplier's guide. The MTT assay was applied according to our previous studies [32-34]. The $\mathrm{IC}_{50}$ values of compounds $5 \mathbf{i}$ and $\mathbf{5 s}$ were calculated from the plots of cell proliferation against concentrations by applying regression analyses on GraphPad Prism Version 5.

2.5. Prediction of ADME Parameters. Physicochemical parameters of synthesized compounds (5a-5s) were calculated by using QikProp 4.8. [35].

2.6. Molecular Docking. A structure based in silico procedure was applied to discover the binding modes of most active compounds $5 \mathbf{i}$ and $\mathbf{5 s}$ to 14 alpha-sterol demethylase enzyme active sites. The crystal structure of enzyme (PDB ID: 1EA1) [36], which was crystallized with the reference drug (fluconazole) of antifungal activity assay, was retrieved from the Protein Data Bank server (http://www.pdb.org).

The structure of ligand was built using the Schrödinger Maestro [37] interface and then was submitted to the Protein Preparation Wizard protocol of the Schrödinger Suite 2016 Update 2 [38]. The ligands were prepared by the LigPrep 3.8 [39] to assign the protonation states at $\mathrm{pH} 7.4 \pm 1.0$ and the atom types, correctly. Bond orders were assigned and hydrogen atoms were added to the structures. The grid generation was formed using Glide 7.1 [40] program and docking runs were performed with single precision docking mode (SP).

\section{Result and Discussion}

3.1. Chemistry. In this study, we synthesized benzimidazoletriazole derivatives (5a-5s) in five steps. The synthetic path of the target compounds was illustrated in Scheme 1.

The structures of the new compounds $(5 \mathbf{a}-\mathbf{5 s})$ were confirmed by FT-IR, ${ }^{1} \mathrm{H}$ NMR, ${ }^{13} \mathrm{C}$ NMR spectroscopy, and mass spectra. The spectroscopic investigations of newly synthesized compounds are accordance with the proposed structure. The IR spectra of the benzimidazole-triazole derivatives (5a-5s) exhibited $\mathrm{N}-\mathrm{H}$ stretching absorption in the region of $3305-2920 \mathrm{~cm}^{-1}$ and $\mathrm{C}=\mathrm{O}$ stretching vibration in the region of $1699-1670 \mathrm{~cm}^{-1}$. The out-of-plane bending of 1,4-disubstituted benzene was assigned at $864-843 \mathrm{~cm}^{-1}$.

${ }^{1} \mathrm{H}$ NMR spectrum showed a broad singlet at $13.32-12.11 \mathrm{ppm}$ due to $\mathrm{NH}$ proton of the benzimidazole ring. The aromatic protons belonging to 4-substituted phenyl gave peaks at $8.02-7.82$ and $8.31-8.39$ as two doublets. Benzimidazole protons were recorded at $7.04-7.67 \mathrm{ppm}$.

In the ${ }^{13} \mathrm{C}$ NMR spectra carbon of $\mathrm{C}=\mathrm{O}$ group was assigned at $190.30-194.94 \mathrm{ppm}$. In the aromatic region, the peaks were seen at estimated areas but the assignments could not be determined, clearly. Chemical shifting of the substituted group and coupling constant of the fluorinated derivatives had coherent results with the theoretical values. HRMS findings were in accordance with the theoretical molecular formula of the compounds (5a-5s).

3.2. Antifungal Activity. The in vitro antifungal screening for all the synthesized compounds was evaluated against Candida glabrata (ATCC 90030), Candida krusei (ATCC 6258), Candida parapsilosis (ATCC 22019), and Candida albicans (ATCC 24433) using twofold serial dilution technique recommended by EUCAST definitive (EDef 7.1) method [29] with the positive control of clinical antifungal drugs ketoconazole and fluconazole. The $\mathrm{MIC}_{50}$ value of the compounds and control drugs are summarized in Table 1.

According to antifungal screening, most of the newly synthesized compounds could effectively inhibit the growth of all the tested fungal strains. All of the final compounds exhibited moderate to good antifungal activities with $\mathrm{MIC}_{50}$ values ranging from 12.5 to $0.78 \mu \mathrm{g} / \mathrm{mL}$. Compounds $5 \mathbf{i}$ and 5s, bearing 3,4-dihydroxyphenyl group, exerted the best activities in inhibiting the growth of all tested fungal strains.

The microbiological results revealed that the antifungal effects of the compounds on Candida species did not depend on N-4 position of triazole. The results show that general structure of the synthesized compounds has an impact on antifungal activity and this effect is further enhanced by the 3,4-dihydroxy structure.

3.3. Inhibition of Ergosterol Biosynthesis. Infections caused by eukaryotic organisms like fungus generally present more difficult treatment problems than those of bacterial infections. There are relatively few antifungal drugs that can identify unique targets not shared with human hosts. The fungal cell wall remains an underdeveloped therapeutic target for selective antifungal agents because of its chitin structure, which is absent in human cells [41, 42]. Most therapies are planned to treat fungal infections and target the ergosterol biosynthesis pathway or its end product, ergosterol, a membrane sterol that is unique to fungi. It is the main sterol and thus is essential for growth and normal membrane function of fungal cell. Besides serving as a bioregulator of membrane fluidity, asymmetry, and integrity, it contributes to proper function of membrane-bound enzymes [43].

The present work is a challenge to understand the probable mechanism of antifungal activity of newly synthesized benzimidazole-triazole hybrid compounds $5 \mathbf{i}$ and $\mathbf{5 s}$. Therefore, we analyzed ergosterol level of C. albicans by LCMSMS studies. Total intracellular sterols were extracted as reported by Breivik and Owades [30]. Ergosterol standard (product number 45480, Sigma-Aldrich, Germany) was used for quantification of ergosterol in both inhibitor-free (negative control) and inhibitor including samples. The most active compounds $5 \mathbf{i}, 5 \mathbf{s}$ and reference drugs were used at $0.78 \mu \mathrm{g} / \mathrm{mL}, 1.56 \mu \mathrm{g} / \mathrm{mL}$, and $3.12 \mu \mathrm{g} / \mathrm{mL}$ concentrations. Ergosterol quantity in negative control samples was regarded as $100 \%$. All concentrations were analyzed in quadruplicate, and the results were expressed as mean \pm standard deviation (SD) [31] (Figure 2).

Ergosterol quantification studies indicated that compounds $5 \mathbf{i}, 5 \mathbf{s}$ and reference agents significantly reduced 
<smiles>COC(=O)c1ccc(C=O)cc1</smiles>

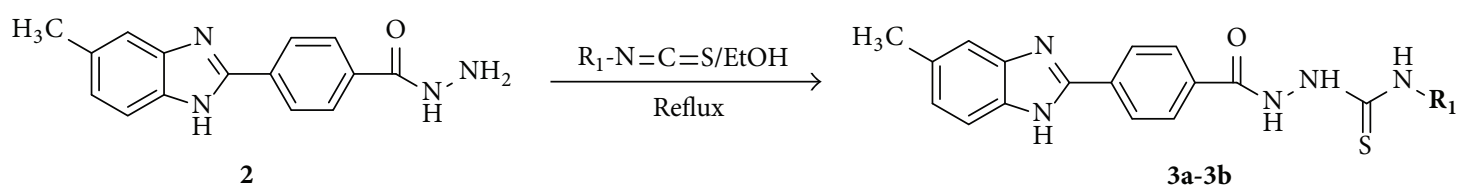

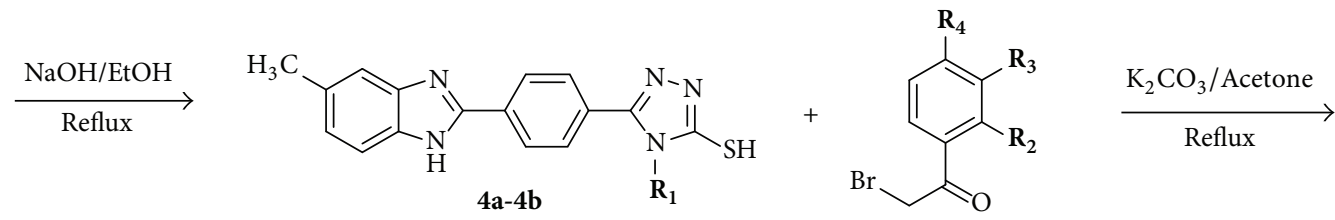<smiles>[R3]c1ccc(C(=O)CSc2nnc(-c3ccc(-c4nc5cc(C)ccc5[nH]4)cc3)n2[R1])c([R2])c1[R15]</smiles>

$\mathbf{R}_{\mathbf{1}}$ : $-\mathrm{CH}_{3},-\mathrm{C}_{2} \mathrm{H}_{5} ; \mathbf{R}_{2}, \mathbf{R}_{3}, \mathbf{R}_{4}$ : - H, -Cl, -F, Br, CN, OH, $\mathrm{CH}_{3}$

\begin{tabular}{ccccc}
\hline Comp. & $\mathbf{R}_{\mathbf{1}}$ & $\mathbf{R}_{\mathbf{2}}$ & $\mathbf{R}_{\mathbf{3}}$ & $\mathbf{R}_{\mathbf{4}}$ \\
\hline $\mathbf{5 a}$ & $-\mathrm{CH}_{3}$ & $-\mathrm{H}$ & $-\mathrm{H}$ & $-\mathrm{H}$ \\
$\mathbf{5 b}$ & $-\mathrm{CH}_{3}$ & $-\mathrm{H}$ & $-\mathrm{H}$ & $-\mathrm{CN}$ \\
$\mathbf{5} \mathbf{c}$ & $-\mathrm{CH}_{3}$ & $-\mathrm{H}$ & $-\mathrm{H}$ & $-\mathrm{F}$ \\
$\mathbf{5 d}$ & $-\mathrm{CH}_{3}$ & $-\mathrm{H}$ & $-\mathrm{H}$ & $-\mathrm{Br}$ \\
$\mathbf{5 e}$ & $-\mathrm{CH}_{3}$ & $-\mathrm{H}$ & $-\mathrm{H}$ & $-\mathrm{CH}_{3}$ \\
$\mathbf{5 f}$ & $-\mathrm{CH}_{3}$ & $-\mathrm{H}$ & $-\mathrm{H}$ & $-\mathrm{Cl}$ \\
$\mathbf{5 g}$ & $-\mathrm{CH}_{3}$ & $-\mathrm{F}$ & $-\mathrm{H}$ & $-\mathrm{F}$ \\
$\mathbf{5 h}$ & $-\mathrm{CH}_{3}$ & $-\mathrm{Cl}$ & $-\mathrm{H}$ & $-\mathrm{Cl}$ \\
$\mathbf{5 i}$ & $-\mathrm{CH}_{3}$ & $-\mathrm{H}$ & $-\mathrm{OH}$ & $-\mathrm{OH}$ \\
$\mathbf{5 j}$ & $-\mathrm{C}_{2} \mathrm{H}_{5}$ & $-\mathrm{H}$ & $-\mathrm{H}$ & $-\mathrm{H}$ \\
$\mathbf{5 k}$ & $-\mathrm{C}_{2} \mathrm{H}_{5}$ & $-\mathrm{H}$ & $-\mathrm{H}$ & $-\mathrm{CN}$ \\
$\mathbf{5 1}$ & $-\mathrm{C}_{2} \mathrm{H}_{5}$ & $-\mathrm{H}$ & $-\mathrm{H}$ & $-\mathrm{F}$ \\
$\mathbf{5 m}$ & $-\mathrm{C}_{2} \mathrm{H}_{5}$ & $-\mathrm{H}$ & $-\mathrm{H}$ & $-\mathrm{Br}$ \\
$\mathbf{5 n}$ & $-\mathrm{C}_{2} \mathrm{H}_{5}$ & $-\mathrm{H}$ & $-\mathrm{H}$ & $-\mathrm{CH}$ \\
$\mathbf{5 0}$ & $-\mathrm{C}_{2} \mathrm{H}_{5}$ & $-\mathrm{H}$ & $-\mathrm{H}$ & $-\mathrm{Cl}$ \\
$\mathbf{5 p}$ & $-\mathrm{C}_{2} \mathrm{H}_{5}$ & $-\mathrm{F}$ & $-\mathrm{H}$ & $-\mathrm{F}$ \\
$\mathbf{5 r}$ & $-\mathrm{C}_{2} \mathrm{H}_{5}$ & $-\mathrm{Cl}$ & $-\mathrm{H}$ & $-\mathrm{Cl}$ \\
$\mathbf{5 s}$ & $-\mathrm{C}_{2} \mathrm{H}_{5}$ & $-\mathrm{H}$ & $-\mathrm{OH}$ & $-\mathrm{OH}$ \\
\hline
\end{tabular}

SCHEME 1: Synthesis way for final compounds.

the level of ergosterol at all tested concentrations. Compound $5 \mathbf{i}$ displayed $61.74 \%, 85.41 \%$, and $93.71 \%$ inhibitions at $0.78 \mu \mathrm{g} / \mathrm{mL}, 1.56 \mu \mathrm{g} / \mathrm{mL}$, and $3.12 \mu \mathrm{g} / \mathrm{mL}$ concentrations, respectively. Inhibitions of $62.05 \%, 84.48 \%$, and $93.12 \%$ were observed for compound $\mathbf{5 s}$ at the same concentrations. Reference drugs ketoconazole and fluconazole indicated similar inhibition potencies to those of compounds $5 \mathbf{i}$ and $5 \mathbf{s}$ in the range of $63.71 \%$ to $94.46 \%$. A concentration-dependent 
TABLE 1: $\mathrm{MIC}_{50}(\mu \mathrm{g} / \mathrm{mL})$ values of compounds $\mathbf{5 a} \mathbf{- 5 s}$.

\begin{tabular}{|c|c|c|c|c|}
\hline Comp. & C. albicans & C. glabrata & C. krusei & C. parapsilosis \\
\hline $5 a$ & 12.5 & 6.25 & 6.25 & 12.5 \\
\hline $5 b$ & 6.25 & 3.12 & 6.25 & 6.25 \\
\hline $5 c$ & 12.5 & 6.25 & 6.25 & 12.5 \\
\hline $5 d$ & 6.25 & 12.5 & 6.25 & 6.25 \\
\hline $5 e$ & 12.5 & 6.25 & 12.5 & 12.5 \\
\hline $5 f$ & 6.25 & 3.12 & 3.12 & 6.25 \\
\hline $5 \mathrm{~g}$ & 3.12 & 6.25 & 6.25 & 6.25 \\
\hline $5 \mathrm{~h}$ & 12.5 & 6.25 & 12.5 & 6.25 \\
\hline $5 i$ & 0.78 & 1.56 & 1.56 & 0.78 \\
\hline $5 \mathbf{j}$ & 12.5 & 6.25 & 12.5 & 12.5 \\
\hline $5 k$ & 12.5 & 6.25 & 12.5 & 12.5 \\
\hline 51 & 6.25 & 12.5 & 6.25 & 12.5 \\
\hline $5 \mathrm{~m}$ & 3.12 & 3.12 & 3.12 & 6.25 \\
\hline $5 n$ & 3.12 & 3.12 & 1.56 & 3.12 \\
\hline 50 & 3.12 & 3.12 & 6.25 & 6.25 \\
\hline $5 p$ & 12.5 & 12.5 & 6.25 & 6.25 \\
\hline $5 r$ & 6.25 & 3.12 & 3.12 & 3.12 \\
\hline $5 s$ & 0.78 & 1.56 & 1.56 & 0.78 \\
\hline Ketoconazole & 0.78 & 1.56 & 1.56 & 1.56 \\
\hline Fluconazole & 0.78 & 1.56 & 1.56 & 0.78 \\
\hline
\end{tabular}

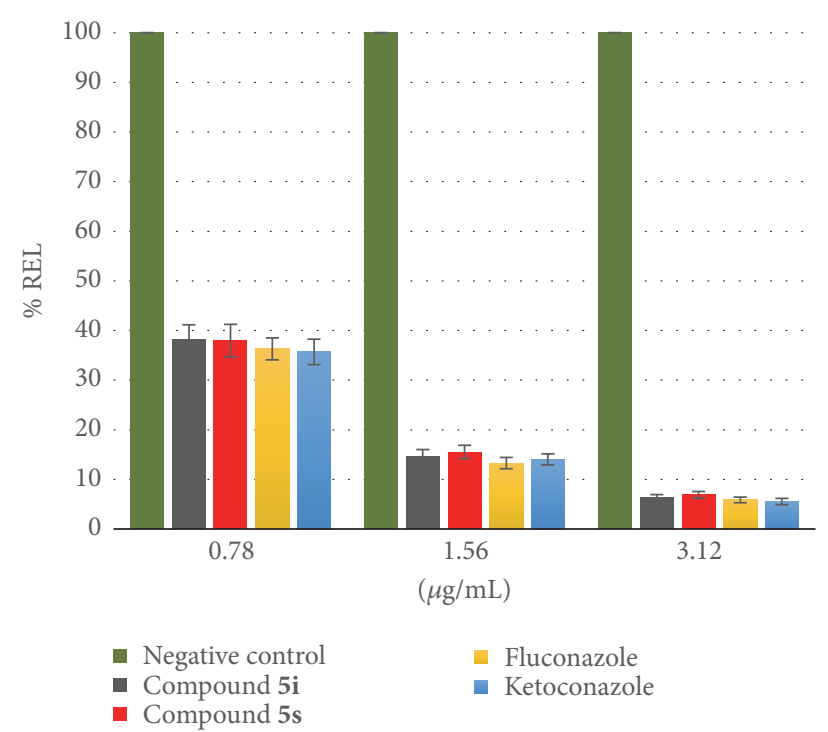

Figure 2: Relative ergosterol level of C. albicans after treatment of synthesized compounds and reference agents.

decrease in the ergosterol level was established for all tested agents. Hence, it can be obviously suggested that compounds $5 \mathbf{i}$ and $5 \mathrm{~s}$ have a role in the ergosterol biosynthesis pathway.

3.4. Cytotoxicity Test. Toxicity is the main reason for the failure at all stages of the new drug development process. The major part of safety-related attrition occurs at preclinical phases while predicting preclinical safety liabilities earlier in the drug development process. This strategy enables the
TABLE 2: $\mathrm{CC}_{50}$ (NIH/3T3), $\mathrm{MIC}_{50}$ (C. albicans), and SI results of selected compounds.

\begin{tabular}{lccc}
\hline Comp. & $\mathrm{CC}_{50}(\mu \mathrm{g} / \mathrm{mL})$ & $\mathrm{MIC}_{50}(\mu \mathrm{g} / \mathrm{mL})$ & $\mathrm{SI}$ \\
\hline $\mathbf{5 i}$ & 86.59 & 0.78 & 111.01 \\
$\mathbf{5 s}$ & 138.85 & 0.78 & 178.01 \\
\hline
\end{tabular}

design and/or selection of improved drug candidates that have more possibilities of becoming commercialized drugs [44]. Therefore, we used the MTT cell viability assay to determine cytotoxicity of the most active compounds $5 \mathbf{i}$ and 5s against NIH/3T3 mouse embryonic fibroblast cell lines (ATCC CRL1658), which is recommended by ISO (10993-5, 2009) [45]. Cytotoxic concentrations (CC) of test compounds are presented as $\mathrm{CC}_{50}$ values in Table 2. Compounds $5 \mathbf{i}$ and $5 \mathrm{~s}$ displayed $\mathrm{CC}_{50}$ of $86.59 \mu \mathrm{g} / \mathrm{mL}$ and $138.85 \mu \mathrm{g} / \mathrm{mL}$, respectively. In order to evaluate selectivity profiles of compounds, selectivity indexes (SIs) were expressed as $\mathrm{CC}_{50}$ $(\mathrm{NIH} / 3 \mathrm{~T} 3) / \mathrm{MIC}_{50}$ (C. albicans). Sis were calculated as 111.01 and 178.01 for compounds $5 \mathbf{i}$ and $\mathbf{5 s}$, respectively. These findings display that the anticandidal activity of the compounds $5 \mathbf{i}$ and $5 \mathrm{~s}$ is not related to general toxicity but can be ascribed to their selective action against Candida species. Thus, cytotoxicity test findings enhanced the importance of compounds $5 \mathbf{i}$ and $\mathbf{5 s}$ as anticandidal drug candidates.

3.5. Theoretical Calculation of ADME Parameters. High biological activity and low toxicological effects are not enough for a compound to become a drug candidate. A good pharmacokinetics profile is also very important for the new drug candidates that should be evaluated earlier in the process 
TABLE 3: Calculated ADME parameters of compounds 5a-5s.

\begin{tabular}{lcccccccccccc}
\hline Comp. & MW & RB & MV & DHB & AHB & PSA & $\log P$ & $\log S$ & PCaco & PM & VRT & VRF \\
\hline $\mathbf{5 a}$ & 439.53 & 4 & 1372.27 & 1 & 5.5 & 83.23 & 5.43 & -7.86 & 754.43 & 2 & 1 & 1 \\
$\mathbf{5 b}$ & 464.54 & 5 & 1438.97 & 1 & 7 & 109.02 & 4.66 & -8.79 & 156.01 & 2 & 0 & 1 \\
$\mathbf{5 c}$ & 457.52 & 4 & 1388.38 & 1 & 5.5 & 83.23 & 5.67 & -8.22 & 754.33 & 2 & 1 & 1 \\
$\mathbf{5 d}$ & 518.43 & 4 & 1425.30 & 1 & 5.5 & 83.23 & 6.00 & -8.72 & 754.38 & 2 & 2 & 1 \\
$\mathbf{5 e}$ & 453.56 & 4 & 1431.21 & 1 & 5.5 & 83.23 & 5.74 & -8.42 & 754.43 & 3 & 1 & 1 \\
$\mathbf{5 f}$ & 473.97 & 4 & 1416.39 & 1 & 5.5 & 83.23 & 5.93 & -8.60 & 754.37 & 2 & 1 \\
$\mathbf{5 g}$ & 508.42 & 4 & 1465.01 & 1 & 5.5 & 80.43 & 6.39 & -9.33 & 740.09 & 2 & 2 \\
$\mathbf{5 h}$ & 475.51 & 4 & 1410.99 & 1 & 5.5 & 82.91 & 5.86 & -8.58 & 643.17 & 2 & 1 \\
$\mathbf{5 i}$ & 471.53 & 6 & 1400.25 & 3 & 7 & 128.41 & 3.79 & -6.86 & 74.95 & 4 & 0 & 1 \\
$\mathbf{5 j}$ & 453.56 & 5 & 1412.00 & 1 & 5.5 & 82.77 & 5.62 & -7.82 & 676.16 & 2 & 1 \\
$\mathbf{5 k}$ & 478.57 & 6 & 1478.70 & 1 & 7 & 108.56 & 4.85 & -8.75 & 139.83 & 2 & 0 & 1 \\
$\mathbf{5 1}$ & 471.55 & 5 & 1428.11 & 1 & 5.5 & 82.77 & 5.86 & -8.18 & 676.08 & 2 & 1 \\
$\mathbf{5 m}$ & 532.46 & 5 & 1465.03 & 1 & 5.5 & 82.77 & 6.19 & -8.68 & 676.13 & 2 & 2 & 1 \\
$\mathbf{5 n}$ & 467.59 & 5 & 1470.94 & 1 & 5.5 & 82.77 & 5.93 & -8.38 & 676.16 & 3 & 1 \\
$\mathbf{5 0}$ & 488.01 & 5 & 1456.12 & 1 & 5.5 & 82.77 & 6.12 & -8.56 & 676.11 & 2 & 1 \\
$\mathbf{5 p}$ & 522.45 & 5 & 1414.92 & 1 & 5.5 & 74.59 & 6.17 & -7.94 & 1124.54 & 2 & 2 \\
$\mathbf{5 r}$ & 489.54 & 5 & 1449.32 & 1 & 5.5 & 80.90 & 6.09 & -8.56 & 712.34 & 2 & 1 \\
$\mathbf{5 s}$ & 485.56 & 7 & 1412.81 & 3 & 7 & 124.13 & 3.93 & -6.33 & 98.80 & 4 & 0 & 1 \\
\hline
\end{tabular}

MW: Molecular weight RB: Number of rotatable bonds MV: Total solvent-accessible volume DHB: Estimated number of hydrogen bond donors AHB: Estimated number of hydrogen bond acceptors PSA: Van der Waals surface area of polar nitrogen and oxygen atoms and carbonyl carbon atoms log $P$ : Predicted octanol/water partition coefficient $\log S$ : Predicted aqueous solubility PCaco: Predicted apparent Caco-2 cell permeability log BB: Predicted brain/blood partition coefficient PM: Number of likely metabolic reactions VRF: Number of violations of Lipinski's rule of five. The rules are: MW $<500, \log P<5, \mathrm{DHB}$ $\leq 5, \mathrm{AHB} \leq 10$, Positive PSA value VRT: Number of violations of Jorgensen's rule of three. The three rules are: $\log S>-5.7, P C a c o>22 \mathrm{~nm} / \mathrm{s}, \mathrm{PM}<7$.

of drug development. Thus, it has become important to examine the ADME profiles of pharmaceuticals as early as possible in the drug discovery process to reduce the amount of wasted time and resources. Moreover, in silico prediction of ADME can be used to identify individual molecules or chemical libraries by evaluating their suitability as potential drug molecules. The determination of the attempted lead optimization before synthesizing the compounds causes less redesign-synthesize-test cycles [46]. Thus, predictions of ADME properties of the all synthesized compounds (5a-5s) were performed by QikProp 4.8 software [35]. This software applies Lipinski's rule of five [47] and Jorgensen's rule of three [48], which evaluate the ADME properties of new drug candidates and are essential for the optimization of a biologically active compound. Molecular weight (MW), number of rotatable bonds (RB), molecular volume (MV), number of hydrogen donors (DHB), number of hydrogen acceptors (AHB), polar surface area (PSA), octanol/water partition coefficient $(\log P)$, aqueous solubility $(\log S)$, apparent Caco-2 cell permeability (PCaco), number of likely primer metabolic reactions (PM), and percent of human oral absorption (\% HOA) are presented in Table 3 along with the violations of rules of three (VRT) and five (VRF). According to Lipinski's rule of five and Jorgensen's rule of three, compounds $5 \mathbf{i}$ and $5 \mathrm{~s}$ are in accordance with the rule by causing no more than one violation. Thus, it can be suggested that the most active anticandidal compounds $5 \mathbf{i}$ and $5 \mathrm{~s}$ may possess a good pharmacokinetic profile, increasing their pharmacological importance.
3.6. Molecular Docking. Docking studies were performed in order to gain more insight into the binding mode of the compounds $5 \mathbf{i}$ and $5 \mathrm{~s}$ to lanosterol $14-\alpha$-demethylase. Due to the fact that lanosterol $14-\alpha$-demethylase is membranebound enzyme, its crystallization from the Candida species is difficult for X-ray analysis and docking studies. Thus, there is no experimental data or crystal structure of this enzyme in Protein Data Bank server. On the other hand, lanosterol 14- $\alpha$-demethylase from Mycobacterium tuberculosis has high homology to that of lanosterol 14- $\alpha$-demethylase from Candida species. It has been reported that these two enzymes have high degree of similarity between the hydrophobic cavities of the catalytic site $[49,50]$. Therefore, docking studies were carried out using X-ray crystal structure of lanosterol 14$\alpha$-demethylase from Mycobacterium tuberculosis in complex with fluconazole (PDB ID: 1EA1) [36] obtained from Protein Data Bank server (http://www.pdb.org).

As stated in antifungal activity section, compounds $\mathbf{5 i}$ and $5 \mathrm{~s}$ were found to be the most active derivatives against $C$. krusei, C. albicans, C. parapsilosis, and C. glabrata with 0.78, $1.56,1.56$, and $0.78 \mu \mathrm{g} / \mathrm{mL} \mathrm{MIC}$ values, respectively. Thus, the main purpose of docking studies was to investigate the possible interaction of these compounds with lanosterol 14$\alpha$-demethylase enzyme.

The two-dimensional (Figures 3(a) and 4(a)) and threedimensional (Figures 3(b) and 4(b)) poses of the compounds $5 \mathbf{i}$ and $5 \mathbf{s}$ are presented in Figures 3 and 4. The docking poses on lanosterol $14-\alpha$-demethylase reveals that the interactions between the compounds 5i, $\mathbf{5 s}$ and HEM450 are very important in terms of binding to active site of enzyme. Totally there 


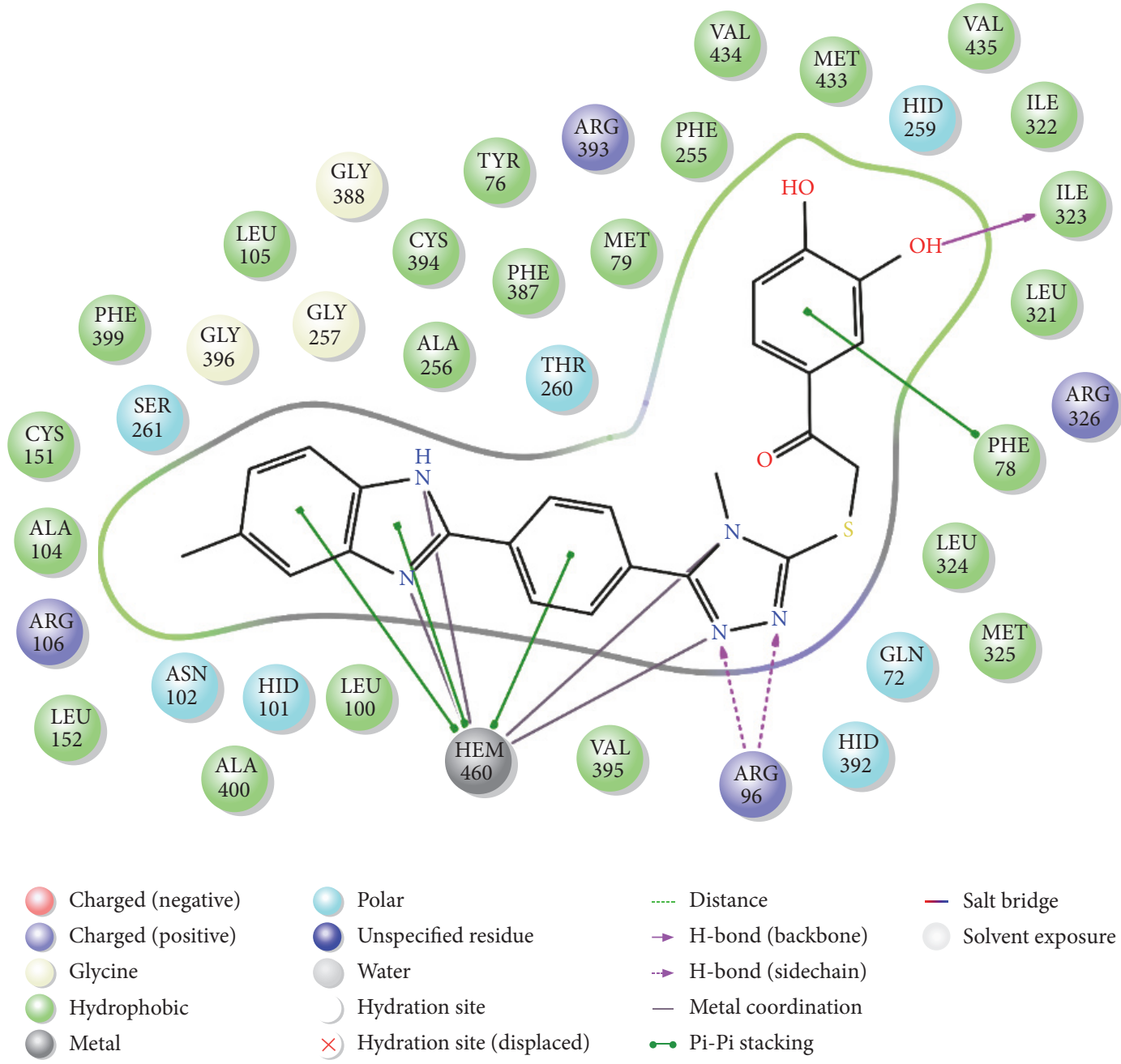

(a)

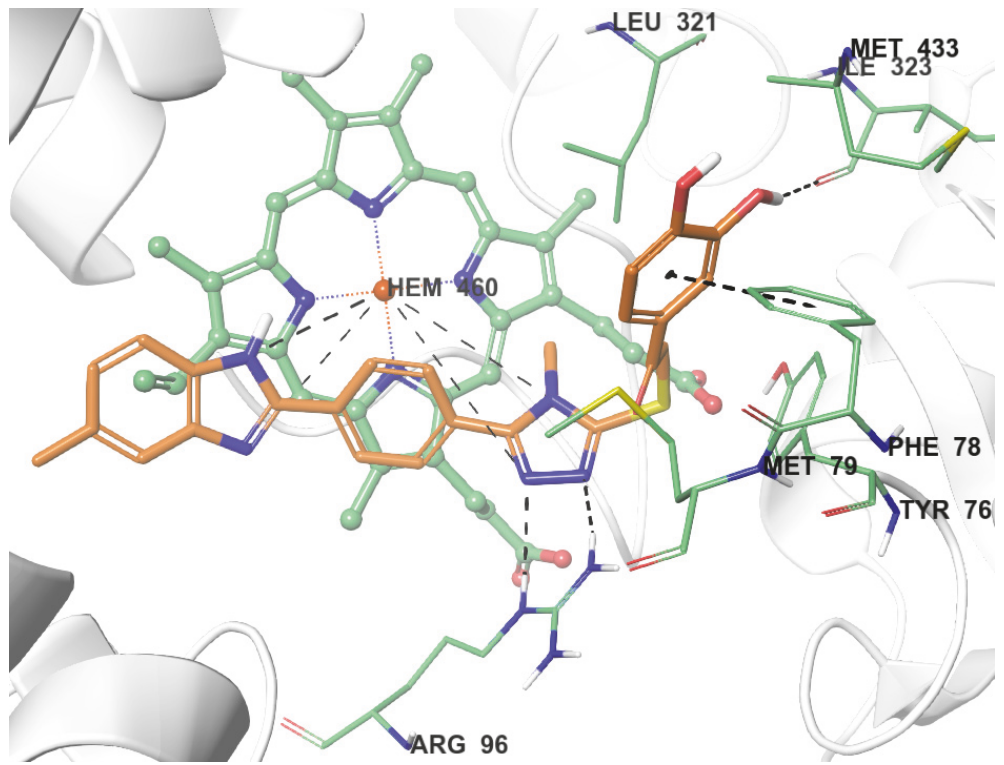

(b)

FIgURE 3: (a) The two-dimensional interacting mode of compound 5i in the active region of lanosterol 14- $\alpha$-demethylase. (b) The threedimensional interacting mode of compound $5 \mathbf{i}$ in the active region of lanosterol 14 - $\alpha$-demethylase. The inhibitor is colored with orange. The HEM molecule is colored green with ball-and-stick model. The binding energy of $\mathbf{5 i}$ is $-81.998 \mathrm{kcal} / \mathrm{mol}$. 


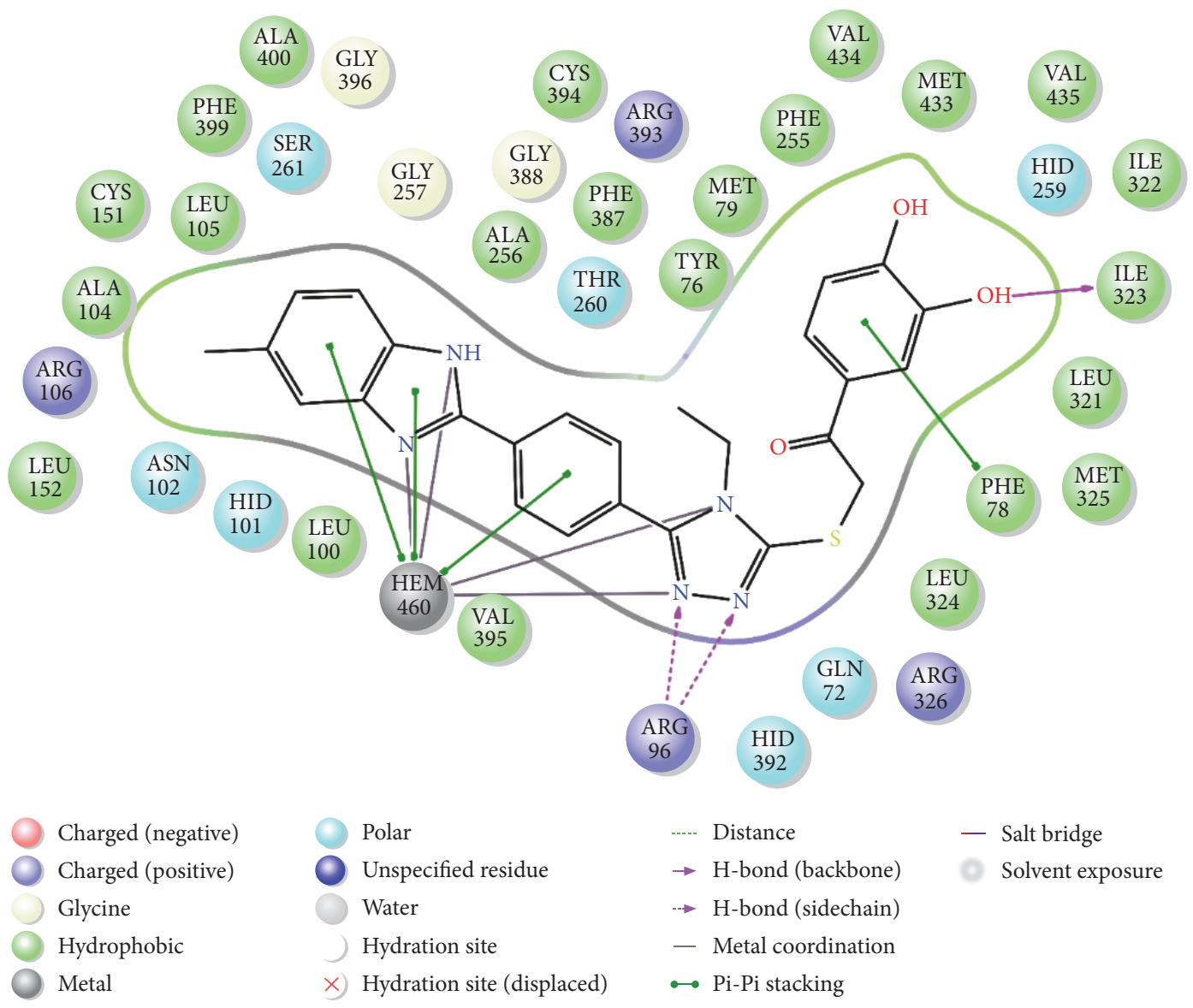

(a)

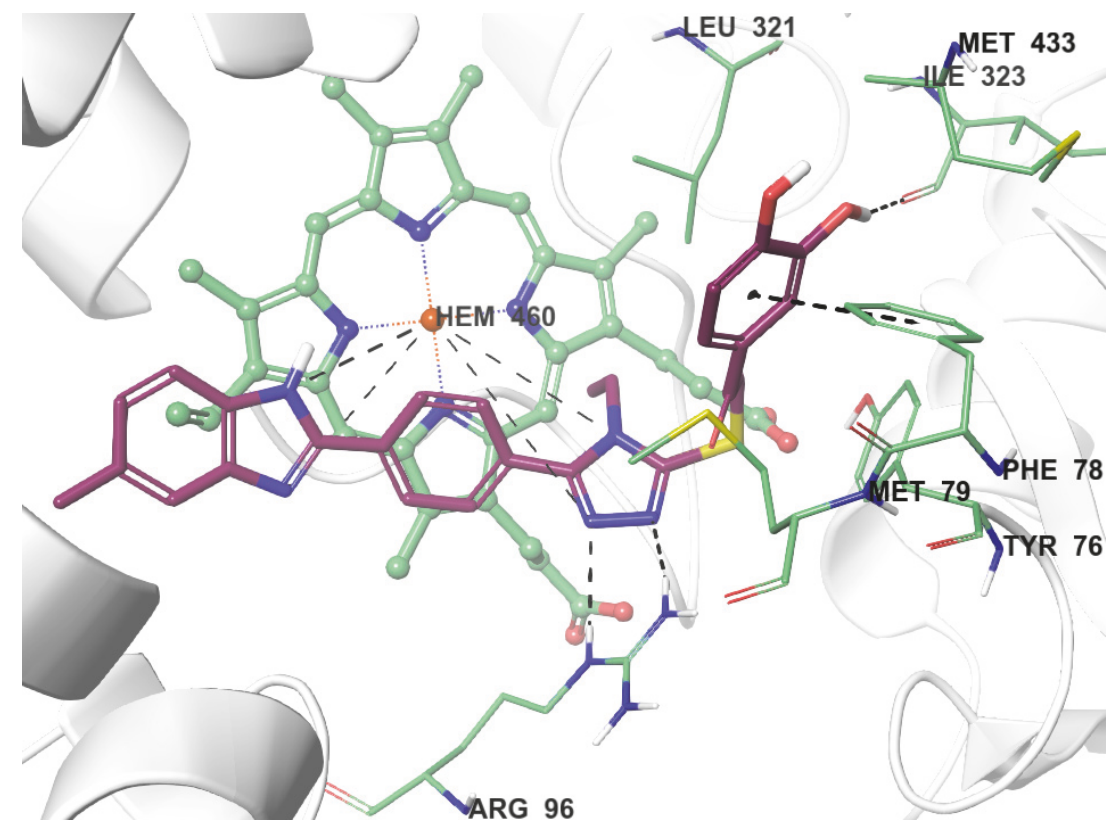

(b)

Figure 4: (a) The two-dimensional interacting mode of compound $5 \mathbf{s}$ in the active region of lanosterol 14- $\alpha$-demethylase. (b) The threedimensional interacting mode of compound $\mathbf{5 s}$ in the active region of lanosterol $14-\alpha$-demethylase. The inhibitor is colored with maroon. The HEM molecule is colored green with ball-and-stick model. The binding energy of $5 \mathrm{~s}$ is $-85.218 \mathrm{kcal} / \mathrm{mol}$. 
are seven interactions between Hem 450 and compounds. Hem 450 creates four metal coordination bonds with the two nitrogen atoms of benzimidazole and two nitrogen atoms triazole (at second and fourth position). Other interactions are seen as $\pi-\pi$ interaction between benzimidazole and phenyl ring (near to benzimidazole) in the structure. In addition, triazole provides binding to active site by forming hydrogen bonds. The nitrogen atoms of triazole (at first and second position) establish two different hydrogen bonds with two neighboring nitrogen atoms in the guanidine moiety of Arg96. Also, phenyl ring at the end of the structure is in interaction with phenyl of Phe78 by doing $\pi-\pi$ interaction.

Synthesized compounds comprise two different substitutions as methyl or ethyl on $\mathrm{N}-1$ position of triazole ring. Looking at activity results and docking studies, it is observed that the methyl or ethyl substituents at N-4 position of triazole do not cause a meaningful difference on biological activity. Furthermore, docking results explain the reason of stronger anticandidal activity of $\mathbf{5 i}$ and $\mathbf{5 s}$. In such compounds, there is an additional interaction at C-3 position of phenyl ring differently common chemical structure. The hydroxyl in this position establishes a hydrogen bond with carbonyl of Ile323. Thus, it can be suggested that C-3 of phenyl is very important in terms of binding to enzyme active site and anticandidal activity. As a result, it is considered that this additional interaction could explain more binding capability and stronger activity of compounds $5 \mathbf{i}$ and $5 s$ than other compounds.

\section{Conclusion}

In the current work, a series of benzimidazole-triazole derivatives (5a-5s) was synthesized and evaluated for their in vitro antifungal activities. Results of antifungal study indicated that compounds $\mathbf{5 i}$ and $\mathbf{5 s}$ have a significant anticandidal activity against all the tested microbial strains. Ergosterol quantification studies using $C$. albicans indicated that compounds $5 \mathbf{i}$ and $5 \mathrm{~s}$ cause a decrease in the level of ergosterol. Since the selectivity of the compounds is a very important parameter to become a good drug candidate, the in vitro toxicity of compounds $\mathbf{5 i}$ and $\mathbf{5 s}$ was evaluated by MTT assay against NIH3T3 cell lines and found as nontoxic at their effective concentrations. ADME prediction and docking studies also enhanced the biological importance of such compounds. As a result it can be declared that synthesized benzimidazoletriazoles are worthwhile to assess in further studies to develop new antifungal agents with higher potency and improved safety profile.

\section{Conflicts of Interest}

The authors declare that there are no conflicts of interest regarding the publication of this paper.

\section{Acknowledgments}

This study was financially supported by Anadolu University Scientific Projects Fund, Project no. 1606S567.

\section{References}

[1] H. Tang, C. Zheng, J. Lv et al., "Synthesis and antifungal activities in vitro of novel pyrazino [2,1-a] isoquinolin derivatives," Bioorganic \& Medicinal Chemistry Letters, vol. 20, no. 3, pp. 979-982, 2010.

[2] W. Wang, C. Sheng, X. Che et al., "Design, synthesis, and antifungal activity of novel conformationally restricted triazole derivatives," Archiv der Pharmazie, vol. 342, no. 12, pp. 732-739, 2009.

[3] H. A. Ghabbour, M. M. Qabeel, W. M. Eldehna, A. Al-Dhfyan, and H. A. Abdel-Aziz, "Design, synthesis, and molecular docking of 1-(1-(4-chlorophenyl)-2-(phenylsulfonyl)ethylidene)-2phenylhydrazine as potent nonazole anticandidal agent," Journal of Chemistry, vol. 2014, Article ID 154357, 2014.

[4] S. Emami, T. Banipoulad, H. Irannejad et al., "Imidazolylchromanones containing alkyl side chain as lanosterol $14 \alpha$ demethylase inhibitors: Synthesis, antifungal activity and docking study," Journal of Enzyme Inhibition and Medicinal Chemistry, vol. 29, no. 2, pp. 263-271, 2014.

[5] R. S. Kankate, P. S. Gide, and D. P. Belsare, "Design, synthesis and antifungal evaluation of novel benzimidazole tertiary amine type of fluconazole analogues," Arabian Journal of Chemistry, 2014.

[6] A. Ahmad, A. Khan, N. Manzoor, and L. A. Khan, "Evolution of ergosterol biosynthesis inhibitors as fungicidal against Candida," Microbial Pathogenesis, vol. 48, no. 1, pp. 35-41, 2010.

[7] C. Sheng, Z. Miao, H. Ji et al., “Three-dimensional model of lanosterol $14 \alpha$-demethylase from Cryptococcus neoformans: Active-site characterization and insights into azole binding," Antimicrobial Agents and Chemotherapy, vol. 53, no. 8, pp. 34873495, 2009.

[8] Q. Zhang, D. Li, P. Wei et al., "Structure-based rational screening of novel hit compounds with structural diversity for cytochrome P450 sterol $14 \alpha$-demethylase from penicillium digitatum," Journal of Chemical Information and Modeling, vol. 50, no. 2, pp. 317-325, 2010.

[9] S. Jacob K, S. Ganguly, P. Kumar, R. Poddar, and A. Kumar, "Homology model, molecular dynamics simulation and novel pyrazole analogs design of Candida albicans CYP450 lanosterol $14 \alpha$-demethylase, a target enzyme for antifungal therapy," Journal of Biomolecular Structure and Dynamics, vol. 35, no. 7, pp. 1446-1463, 2017.

[10] İ. S. Doğan, S. Saraç, S. Sari et al., "New azole derivatives showing antimicrobial effects and their mechanism of antifungal activity by molecular modeling studies," European Journal of Medicinal Chemistry, vol. 130, pp. 124-138, 2017.

[11] K. K. Reddy, S. K. Singh, S. K. Tripathi, C. Selvaraj, and V. Suryanarayanan, "Shape and pharmacophore-based virtual screening to identify potential cytochrome P450 sterol 14 $\alpha$-demethylase inhibitors," Journal of Receptors and Signal Transduction, vol. 33, no. 4, pp. 234-243, 2013.

[12] A. Stana, D. C. Vodnar, R. Tamaian et al., "Design, synthesis and antifungal activity evaluation of new thiazolin-4-ones as potential lanosterol $14 \alpha$-demethylase inhibitors," International Journal of Molecular Sciences, vol. 18, no. 1, article no. 177, 2017.

[13] A. G. S. Warrilow, J. G. L. Mullins, C. M. Hull et al., "S279 point mutations in Candida albicans sterol $14-\alpha$ demethylase (CYP51) reduce in vitro inhibition by fluconazole," Antimicrobial Agents and Chemotherapy, vol. 56, no. 4, pp. 2099-2107, 2012.

[14] A. A. Sagatova, M. V. Keniya, R. K. Wilson, M. Sabherwal, J. D. A. Tyndall, and B. C. Monk, "Triazole resistance mediated by 
mutations of a conserved active site tyrosine in fungal lanosterol 14 $\alpha$-demethylase," Scientific Reports, vol. 6, Article ID 26213, pp. $1-6,2016$.

[15] C. Sheng, W. Zhang, M. Zhang et al., "Homology modeling of lanosterol $14 \alpha$-demethylase of Candida albicans and Aspergillus fumigatus and insights into the enzyme-substrate interactions," Journal of Biomolecular Structure and Dynamics, vol. 22, no. 1, pp. 91-99, 2004.

[16] Y. Jiang, J. Zhang, Y. Cao et al., "Synthesis, in vitro evaluation and molecular docking studies of new triazole derivatives as antifungal agents," Bioorganic \& Medicinal Chemistry Letters, vol. 21, no. 15, pp. 4471-4475, 2011.

[17] D. J. Sheehan, C. A. Hitchcock, and C. M. Sibley, "Current and emerging azole antifungal agents," Clinical Microbiology Reviews, vol. 12, no. 1, pp. 40-79, 1999.

[18] A. A.-M. Abdel-Hafez, "Benzimidazole condensed ring systems: New synthesis and antineoplastic activity of substituted 3,4-dihydro- and 1,2,3,4-tetrahydro-benzo[4,5]imidazo[1, 2a]pyrimidine derivatives," Archives of Pharmacal Research, vol. 30, no. 6, pp. 678-684, 2007.

[19] Z. Ateş-Alagöz, C. Kuş, and T. Çoban, "Synthesis and antioxidant properties of novel benzimidazoles containing substituted indole or 1,1,4,4-tetramethyl-1,2,3,4-tetrahydro-naphthalene fragments," Journal of Enzyme Inhibition and Medicinal Chemistry, vol. 20, no. 4, pp. 325-331, 2005.

[20] N. N. Gülerman, H. N. Doan, S. Rollas, C. Johansson, and C. Çelik, "Synthesis and structure elucidation of some new thioether derivatives of 1,2,4-triazoline-3-thiones and their antimicrobial activities," II Farmaco, vol. 56, no. 12, pp. 953-958, 2001.

[21] F. Hernández-Luis, A. Hernández-Campos, R. Castillo et al., "Synthesis and biological activity of 2-(trifluoromethyl)$1 \mathrm{H}$-benzimidazole derivatives against some protozoa and Trichinella spiralis," European Journal of Medicinal Chemistry, vol. 45, no. 7, pp. 3135-3141, 2010.

[22] K. S. Jain, V. M. Khedkar, N. Arya, P. V. Rane, P. K. Chaskar, and E. C. Coutinho, "Design, synthesis \& evaluation of condensed $2 \mathrm{H}$-4-arylaminopyrimidines as novel antifungal agents," European Journal of Medicinal Chemistry, vol. 77, pp. 166-175, 2014.

[23] H.-T. Le, I. B. Lemaire, A.-K. Gilbert et al., "Histograninlike antinociceptive and anti-inflammatory derivatives of ophenylenediamine and benzimidazole," The Journal of Pharmacology and Experimental Therapeutics, vol. 309, no. 1, pp. 146$155,2004$.

[24] A. T. Mavrova, P. Denkova, Y. A. Tsenov, K. K. Anichina, and D. I. Vutchev, "Synthesis and antitrichinellosis activity of some bis(benzimidazol-2-yl)amines," Bioorganic \& Medicinal Chemistry, vol. 15, no. 18, pp. 6291-6297, 2007.

[25] J. R. Maxwell, A. C. Wasdahl, D. A. Wolfson, and V. I. Stenberg, "Synthesis of 5-aryl-2H-tetrazoles, 5-aryl-2H-tetrazole-2-acetic acids, and [(4-phenyl-5-aryl-4H-1,2,4-triazol-3-yl)thio]acetic acids as possible superoxide scavengers and antiinflammatory agents," Journal of Medicinal Chemistry, vol. 27, no. 12, pp. 15651570, 1984.

[26] Y. Özkay, Y. Tunali, H. Karaca, and I. Işikdağ, "Antimicrobial activity and a SAR study of some novel benzimidazole derivatives bearing hydrazone moiety," European Journal of Medicinal Chemistry, vol. 45, no. 8, pp. 3293-3298, 2010.

[27] H.-Z. Zhang, G. L. V. Damu, G.-X. Cai, and C.-H. Zhou, "Design, synthesis and antimicrobial evaluation of novel benzimidazole type of Fluconazole analogues and their synergistic effects with Chloromycin, Norfloxacin and Fluconazole," European Journal of Medicinal Chemistry, vol. 64, pp. 329-344, 2013.

[28] G. L. V. Damu, Q. Wang, H. Zhang, Y. Zhang, J. Lv, and C. Zhou, "A series of naphthalimide azoles: design, synthesis and bioactive evaluation as potential antimicrobial agents," Science China Chemistry, vol. 56, no. 7, pp. 952-969, 2013.

[29] EUCAST, Definitive Document EDef 7.1: Method for the Determination of Broth Dilution MICs of Antifungal Agents for Fermentative Yeasts.

[30] O. N. Breivik and J. L. Owades, "Yeast analysis, spectrophotometric semimicrodetermination of ergosterol in yeast," Journal of Agricultural and Food Chemistry, vol. 5, no. 5, pp. 360-363, 1957.

[31] H. K. Gençer, U. A. Çevik, S. Levent et al., "New benzimidazole1,2,4-triazole hybrid compounds: synthesis, anticandidal activity and cytotoxicity evaluation," Molecules, vol. 22, no. 4, article 507, 507 pages, 2017.

[32] N. Ö. Can, D. Osmaniye, S. Levent et al., "Synthesis of new hydrazone derivatives for MAO enzymes inhibitory activity," Molecules, vol. 22, no. 8, p. 1381, 2017.

[33] Ü. Demir Özkay, Ö. D. Can, B. N. Sağlik et al., "Design, synthesis, and AChE inhibitory activity of new benzothiazolepiperazines," Bioorganic \& Medicinal Chemistry Letters, vol. 26, no. 22, pp. 5387-5394, 2016.

[34] B. N. Sağlık, S. Ilgın, and Y. Özkay, "Synthesis of new donepezil analogues and investigation of their effects on cholinesterase enzymes," European Journal of Medicinal Chemistry, vol. 124, pp. 1026-1040, 2016.

[35] QikProp Schrödinger, LLC, New York, NY, USA, 2016, version 4.8.

[36] L. M. Podust, T. L. Poulos, and M. R. Waterman, "Crystal structure of cytochrome P450 14 $\alpha$-sterol demethylase (CYP51) from Mycobacterium tuberculosis in complex with azole inhibitors," in Proceedings of the National Academy of Sciences, vol. 98, pp. 3068-3073, 2001.

[37] Maestro, version 10.6, Schrödinger, LLC, New York, NY, USA, 2016.

[38] Schrödinger, LLC, New York, NY, USA, 2016.

[39] LigPrep Schrödinger, LLC, New York, NY, USA, 2016, version 3.8.

[40] Glide Schrödinger, LLC, New York, NY, USA, 2016, version 7.1.

[41] M. A. Malik, S. A. Al-Thabaiti, and M. A. Malik, "Synthesis, structure optimization and antifungal screening of novel tetrazole ring bearing acyl-hydrazones," International Journal of Molecular Sciences, vol. 13, no. 9, pp. 10880-10898, 2012.

[42] S. Dhingra and R. A. Cramer, "Regulation of sterol biosynthesis in the human fungal pathogen Aspergillus fumigatus: Opportunities for therapeutic development," Frontiers in Microbiology, vol. 8, article no. 92, 2017.

[43] A. Lupetti, R. Danesi, M. Campa, M. D. Tacca, and S. Kelly, "Molecular basis of resistance to azole antifungals," Trends in Molecular Medicine, vol. 8, no. 2, pp. 76-81, 2002.

[44] J. A. Kramer, J. E. Sagartz, and D. L. Morris, “The application of discovery toxicology and pathology towards the design of safer pharmaceutical lead candidates," Nature Reviews Drug Discovery, vol. 6, no. 8, pp. 636-649, 2007.

[45] International Organization for Standardization, Biological Evaluation of Medical Devices-Part 5: Tests for In Vitro cytotoxicity ISO-10993-5, 3rd edition, 2009.

[46] D. Butina, M. D. Segall, and K. Frankcombe, "Predicting ADME properties in silico: Methods and models," Drug Discovery Therapy, vol. 7, no. 11, pp. S83-S88, 2002. 
[47] C. A. Lipinski, F. Lombardo, B. W. Dominy, and P. J. Feeney, "Experimental and computational approaches to estimate solubility and permeability in drug discovery and development settings," Advanced Drug Delivery Reviews, vol. 46, no. 1-3, pp. 3-26, 2001.

[48] W. L. Jorgensen and E. M. Duffy, "Prediction of drug solubility from structure," Advanced Drug Delivery Reviews, vol. 54, no. 3, pp. 355-366, 2002.

[49] R. González-Chávez, R. Martínez, M. E. Torre-Bouscoulet, M. Gallo, and M. M. González-Chávez, "De novo design of noncoordinating indolones as potential inhibitors for lanosterol 14$\alpha$-demethylase (CYP51)," Chemical \& Pharmaceutical Bulletin, vol. 62, no. 1, pp. 16-24, 2014.

[50] R. Gollapudy, S. Ajmani, and S. A. Kulkarni, "Modeling and interactions of aspergillus fumigatus lanosterol 14- $\alpha$ demethylase 'A' with azole antifungals," Bioorganic \& Medicinal Chemistry, vol. 12, no. 11, pp. 2937-2950, 2004. 

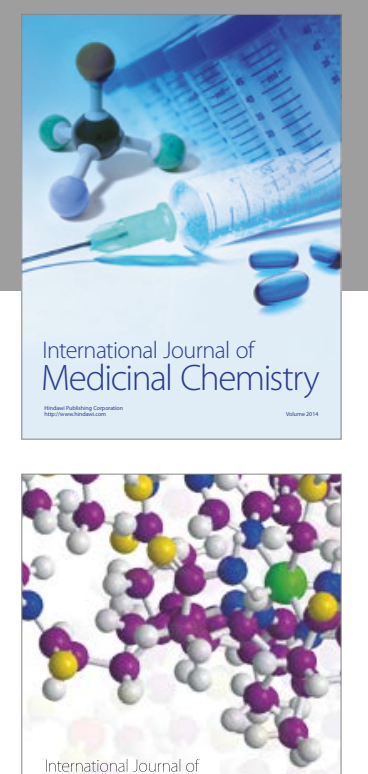

Carbohydrate Chemistry

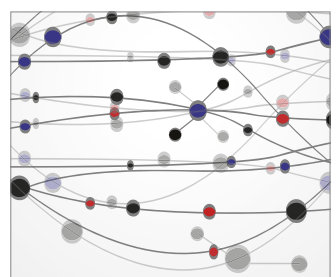

The Scientific World Journal
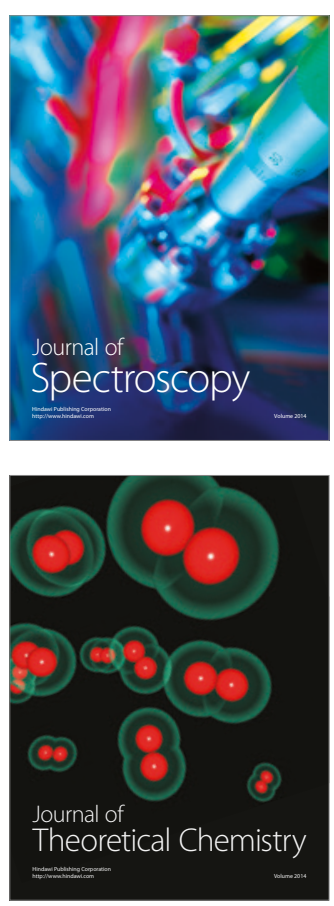
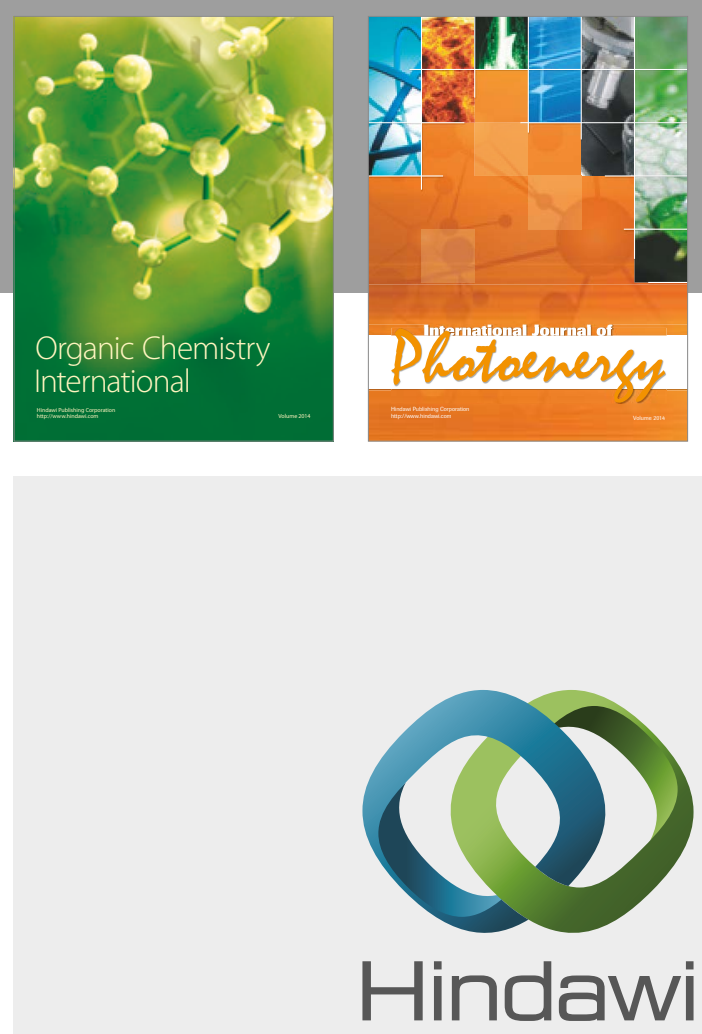

Submit your manuscripts at

https://www.hindawi.com

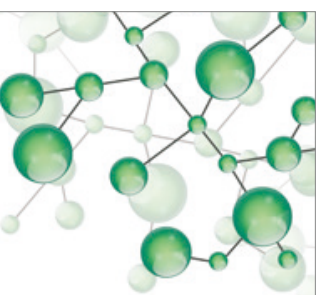

International Journal of

Inorganic Chemistry

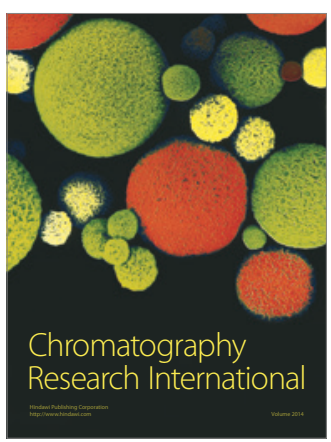

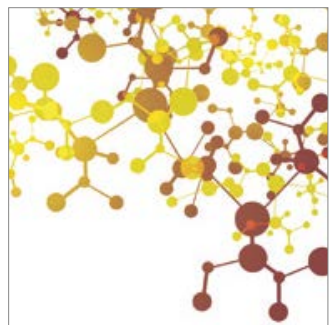

Applied Chemistry
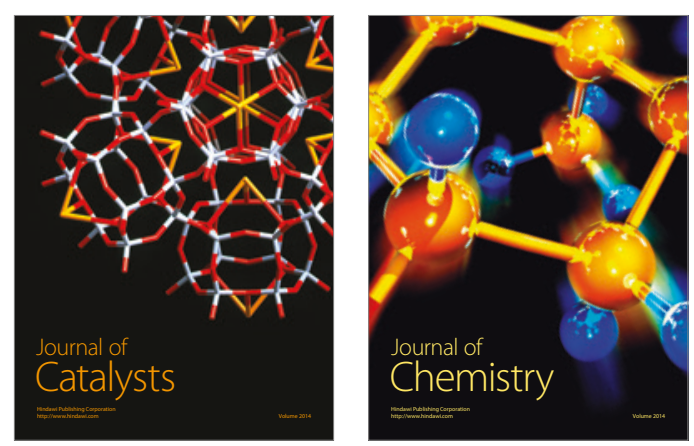
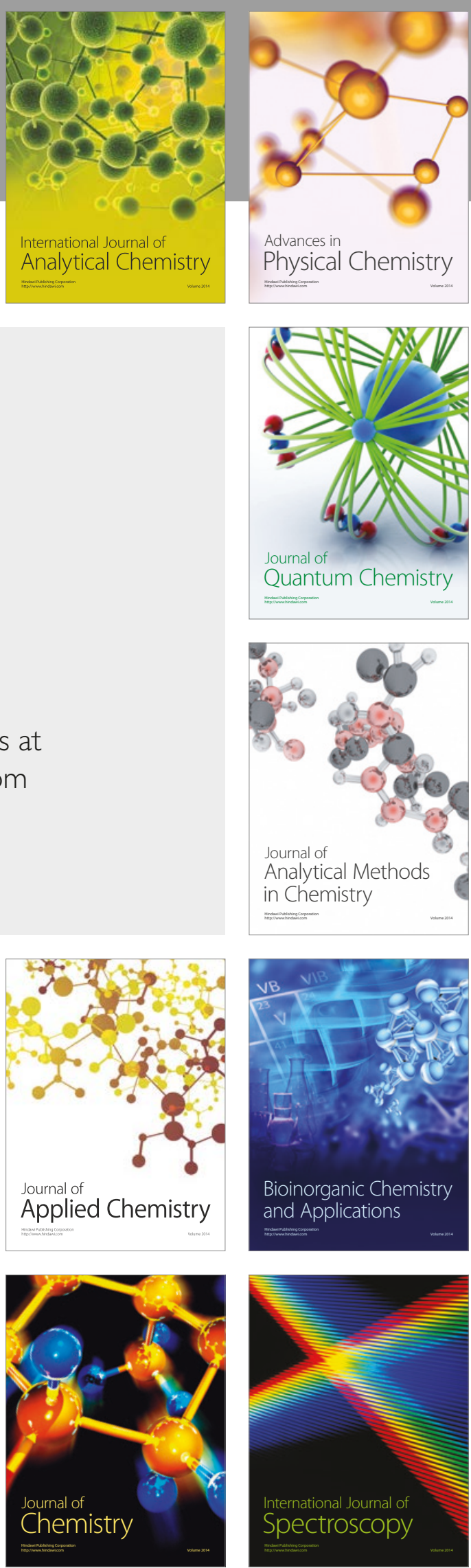